\title{
Atomistically informed dislocation dynamics in fec crystals
}

\author{
E. Martínez , J. Marian , A. Arsenlis , M. Victoria , J.M. Perlado \\ Chemistry and Materials Science Directorate, Lawrence Livermore National Laboratory, Livermore, CA 94550, USA \\ Instituto de Fusión Nuclear, Universidad Politécnica de Madrid, 28006 Madrid, Spain
}

\begin{abstract}
We develop a nodal dislocation dynamics (DD) model to simulate plastic processes in fec crystals. The model explicitly accounts for all slip systems and Burgers vectors observed in fec systems, including stacking faults and partial dislocations. We derive simple conservation rules that describe all partial dislocation interactions rigorously and allow us to model and quantify cross-slip processes, the structure and strength of dislocation junctions, and the formation of fcc-specific structures such as stacking fault tetrahedra. The DD framework is built upon isotropic non-singular linear elasticity and supports itself on information transmitted from the atomistic scale. In this fashion, connection between the meso and micro scales is attained self-consistently, with all material parameters fitted to atomistic data. We perform a series of targeted simulations to demonstrate the capabilities of the model, including dislocation reactions and dissociations and dislocation junction strength. Additionally we map the four-dimensional stress space relevant for cross-slip and relate our findings to the plastic behavior of monocrystalline fce metals.
\end{abstract}

Keywords: Dislocation dynamics; fec plasticity; Cross-slip; Dislocation locks; Numerical methods

\section{Introduction}

Crystal plasticity in deformed materials is governed by the collective behavior of large ensembles of dislocations. Although continuum laws based on effective dislocation densities can be formulated to describe the macroscopic material response under a variety of loading conditions, dislocation motion and interactions are heterogeneous phenomena that display an intricate dependence on the underlying microstructure. The details of these interactions, which are often important in many scenarios, are lost in continuum models based on average dislocation densities. In this sense, atomistic methods have been profusely utilized in recent times to study isolated dislocation interaction mechanisms. However, they suffer from space and time scale limitations and therefore fail to properly capture the long-range character of dislocation stress fields, let alone the statistical nature of crystal plasticity. Alternatively, dislocation dynamics (DD) is a direct approach that attempts to simulate the aggregate behavior of large dislocation ensembles at the mesoscale by decomposing 
dislocation lines of arbitrary curvature and character into piecewise segments (Kubin et al., 1992; Devincre and Kubin, 1997; Kubin et al., 1998; Zbib et al., 1998; Schwarz, 1999; Ghoniem and Sun, 1999; Zbib et al., 2000; Bulatov et al., 2001; Cai et al., 2004). Nevertheless, the number of segments, $N$, can get quite large $\left(\sim 10^{6}-10^{8}\right)$ for meaningful simulations, and the computation of long-range forces is an $\mathcal{O}\left(N^{2}\right)$ problem that can become computationally intensive for large systems. For an excellent review on these and more aspects of DD see Zbib and Diaz de la Rubia (2002) and references therein.

The application of DD to model one aspect or another of crystal plasticity in fcc metals in 3D started in the late 1980s with the pioneering work of Kubin and collaborators (Lepinoux and Kubin, 1987; Kubin et al., 1992) and Ghoniem et al. (Ghoniem and Amodeo, 1988; Amodeo and Ghoniem, 1991). However, it has not been until recently that detailed studies involving complex geometries and relatively large dislocation densities have been undertaken (Verdier et al., 1998; Shenoy et al., 2000; Shin et al., 2001; Dupuy and Fivel, 2002; Madec et al., 2002; Kubin et al., 2003; Depres et al., 2003; Shehadeh et al., 2005; Devincre et al., 2006). Most of these works focus on mapping the strength of dislocation junctions as a function of the reacting geometry, in a clear attempt to capture the elementary mechanisms attendant to forest hardening in stage II of fcc deformation. Nevertheless, with the notable exception of the work by Shenoy et al. (2000) and Shin et al. (2001), and the two-dimensional studies by Hardikar et al. (2001) and Mohles $(2001,2002)$, there have not been many DD studies where the extended nature of dislocations in fcc materials has been explicitly taken into account. ${ }^{1}$ However, important details of dislocation junction formation may be overlooked by using models that neglect dislocation dissociation in fce crystals. In addition, several aspects of dislocation cross-slip, relevant to stage III plasticity, which are non-trivial in fcc metals, may not be properly described using traditional cross-slip models. Furthermore, the theoretical framework of most dislocation dynamics tools is based on isotropic linear elasticity, which ignores core effects despite the fact that it has recently been shown that they govern important aspects of dislocation processes (Vitek, 1985; Justo et al., 1997), and that the core cut-off radius is not a universal parameter and may affect the calculation of dislocation energies (LeSar, 2004; Lothe and Hirth, 2005). Another important reason to consider partial dislocation reactions is the study of irradiation defects, such as stacking fault tetrahedra (SFT), and their interactions with dislocations. Often, defects created in farfrom-equilibrium conditions in fcc systems (e.g. irradiation, quenching, etc.) will not generally be expressible in terms of perfect dislocations, which precludes the use of DD techniques that do not contain this level of detail.

For the reasons outlined above, in this work we set out to provide a dislocation dynamics methodology that accounts for perfect dislocation dissociation and incorporates atomistic information regarding core sizes and energies. Our methodology hinges on a novel non-singular linear elasticity formulation, whose free parameters are fitted to carefully designed atomistic simulations. This article is organized as follows: in Section 2, we describe the existing DD framework upon which we build our methodology, we present the new theoretical developments incorporated in DD to treat dislocation partials, and explain the atomistics-based fitting procedure; next, in Section 3, we apply our method to several well-studied plastic phenomena in fcc metals, including dislocation junctions, cross-slip, and the formation of SFTs, and discuss the results; finally, in Section 4 we present our conclusions.

\section{Methodology}

This section comprises four main parts. First, we give a brief overview of the mean features of the existing DD methodology. Second, we show the theoretical developments leading to new conservation rules for the introduction of partial dislocations. We then propose a new algorithm for treating complex multinodes, which are bound to appear when partial dislocations are present. Finally, we fit the adjustable parameters of our DD methodology to results of Shockley partial equilibrium spacings obtained with molecular statics (MS), which here acts as our first-principles technique. 


\subsection{Underlying DD model}

\subsubsection{Non-singular continuum theory of dislocations}

The linear elastic formulation used in our DD method is the non-singular theory derived by Cai et al. (2006). The singularity intrinsic to the classical continuum theory is removed in this formulation by spreading the Burgers vector isotropically about every point on the dislocation line using a spreading function characterized by a single parameter $a$, the so-called core width. A particular form of the spreading function chosen in this formulation leads to simple analytic formulations for the stress produced by straight dislocation segments, the segment self- and interaction energies, and the forces on the segments. For any value $a>0$, the total energy and the stress remain finite everywhere and, what is more, the well-known singular expressions are recovered for $a=0$. Additionally, the formulation is self-consistent in the sense that the expressions for the force obtained by direct differentiation of the non-singular energy and by recurring to the Peach-Köhler formula are identical. The value of $a$ partitions the energy of a dislocation between its elastic (long-range) and core (short-range) contributions. $a$ ought to be chosen so as to make this partition as faithful to the atomistic description as necessary. For example, below we match the atomistic and continuum energies of a given dislocation configuration to obtain a general value for our simulations. In this fashion, we can then compute core energies and include core effects during dislocation reactions, an issue largely ignored to date in DD simulations. However, in principle the value of $a$ depends upon the Burgers vector and the character of a dislocation (Lothe and Hirth, 2005), something that is not taken into account in the present formulation (there is no analytical theory at present that deals with this shortcoming). In any case, the general solution for a single $a$ still has a clear connection to more fundamental, atomistic models of dislocations, as we shall show below.

\subsubsection{Brief overview of ParaDiS}

ParaDiS, and its companion serial version DDLab, are the fruits of a sustained effort at Lawrence Livermore National Laboratory to develop a massively parallel three-dimensional DD methodology specifically designed for investigating the collective behavior of large numbers of dislocations (Bulatov et al., 2004; Arsenlis et al., 2007). The dislocation ensemble is replaced by a network of nodes with the appropriate connectivity, which act as pointwise limits of each of the discretized segments. Each segment carries a unit of 'vector current' or Burgers vector, which denotes the direction and magnitude of the displacement carried by the dislocation as it moves. Each segment is assigned a line tangent, given by the unit vector connecting the two segment nodes. The nodes move in response to the local stress tensor as dictated by the mobility law, which essentially translates the local force exerted on each node into the corresponding nodal velocities. In addition to moving the nodes, ParaDis evolves the network topology to reflect the physics of dislocation motion and collisions in real crystals. Handling the evolving topology of moving and intersecting lines is a daunting bookkeeping task, especially in a parallel implementation. It is therefore highly desirable to keep the logical complexity of the topological switches to a minimum. Presently, ParaDis relies on two basic operations: (i) insertion of new nodes, and (ii), merging two nodes into one. Even though topological changes consume only a small fraction of the computing time, the associated logic and bookkeeping constitute upwards of $50 \%$ of the ParaDis source code.

\subsection{Implementation of partial dislocations}

From a topological point of view, the dissociation of a (perfect) dislocation can be achieved by using a simple node-splitting scheme. Splitting highly connected dislocation nodes is one of the most commonly used topological changes in discrete DD methods. However, the explicit introduction of partial dislocations in an fcc-DD model presents two main difficulties which are not generally encountered in the modeling of bcc or other systems. First, dislocation dissociation is governed by Frank's rule, which is an energy criterion that relies solely on the crystal's geometry and the stacking fault energy. However, the direct consideration of the system energetics is generally not a structural part of discrete DD models, where forces are calculated directly from the applied stresses through the well-known Peach-Köhler formula. Thus, one must resort to splitting criteria based on power dissipation, which can be calculated without the explicit knowledge of the system 
energetics. In our model, the energy per unit time dissipated by the forces acting on a specific dislocation node $n_{i}$ is calculated as $\dot{W}_{i}=F_{i} v_{i}$, where $F_{i}$ and $v_{i}$ are the total force and the velocity acting on node $i$. The power dissipation criterion states that a new state $\mathscr{S}^{\prime}$ will be preferred over the current state $\mathscr{S}$ if and only if $\Delta \dot{W}=\dot{W}^{\prime}-\lambda \dot{W}>0$, where $\lambda>1$ is an adjustable parameter that reflects the inertial resistance of a dislocation node to change its current state. In other words, splitting will spontaneously occur only if more energy per unit time is dissipated by undergoing the topological change than by remaining in the (biased) present state. Here we have taken a conservative value of $\lambda=1.01$ for all our dislocation simulations.

Secondly, the treatment of partial dislocations in $\mathrm{fcc}$ metals introduces the need to account for stacking faults, characterized by the stacking fault energy, $\gamma$. Although stacking faults are inherent to all partial dislocations, for clarity we derive all expressions for Shockley partials and then provide suitable generalizations for the rest.

$\gamma$ dictates the equilibrium separation distance between adjoining Shockley partials by balancing the elastic repulsion with the increase in system energy due to the stacking fault area. In a force method such as DD we need to express this interaction in terms of the appropriate force $\mathbf{F}_{\mathrm{SF}}$. To derive an expression for $\mathbf{F}_{\mathrm{SF}}$ we consider the configuration shown in Fig. 1, with two Shockley partial dislocation segments of arbitrary curvature separated by a distance (stacking fault width) of $\left(r_{2}-r_{1}\right)$. $\mathbf{F}_{\mathrm{SF}}$ for each segment is then

$$
\begin{aligned}
& E_{\mathrm{SF}}=\gamma A, \\
& \mathbf{F}_{\mathrm{SF}_{1}}=-\frac{\partial E_{\mathrm{SF}}}{\partial r_{1}} \mathbf{u}_{r_{1}}=\gamma \theta r_{1} \mathbf{u}_{r_{1}}=\gamma \ell_{1} \mathbf{u}_{r_{1}}, \\
& \mathbf{F}_{\mathrm{SF}_{2}}=-\frac{\partial E_{\mathrm{SF}}}{\partial r_{2}} \mathbf{u}_{r_{2}}=-\gamma \theta r_{2} \mathbf{u}_{r_{2}}=-\gamma \ell_{2} \mathbf{u}_{r_{2}},
\end{aligned}
$$

where $A$ is the area enclosed between the two dislocation segments (see Fig. 1). This means that the force per unit dislocation length, $f_{\mathrm{SF}}$, as we can see, is constant regardless of the partial separation distance, i.e. $F_{\mathrm{SF}_{1}} / \ell_{1}=F_{\mathrm{SF}_{2}} / \ell_{2}=f_{\mathrm{SF}}=\gamma$.

However, contrary to the example shown in Fig. 1, in our simulations there is no explicit labeling of leading and trailing partials and, thus, the area enclosed between them is not defined. Hence, one complication remains, that is, to ensure that the force due to the stacking fault always point towards the other partial, i.e. as Eqs. (1) show, the stacking fault force must be self-contained. In addition, the force due to the stacking fault must act on the plane where the stacking fault is defined (\{ $\left.\begin{array}{lll}1 & 1 & 1\end{array}\right\}$-type planes in fcc metals). Thus, $\mathbf{f}_{\mathrm{SF}}$ must be locally orthogonal to both the line tangent $\xi$ and the slip plane normal $\mathbf{n}$. Also, as discussed above, $\mathbf{f}_{\mathrm{SF}}$ must be directly proportional to $\gamma$. The expression that compounds these requirements is

$$
\mathbf{f}_{\mathrm{SF}}=\boldsymbol{\xi} \times \gamma \mathbf{n}
$$

To ensure $f_{\mathrm{SF}}=\gamma$, both $\mathbf{n}$ and $\boldsymbol{\xi}$ must be unit vectors. This fixes $\mathbf{n}=\left\langle\frac{1}{\sqrt{3}} \frac{1}{\sqrt{3}} \frac{1}{\sqrt{3}}\right\rangle$ for Shockley partial dislocations. Below we will show that the magnitude of $\gamma \mathbf{n}$ is dislocation dependent, and need not be a unit vector, nor does it need to correspond to a given slip plane in particular. Therefore, we hereon assign a new

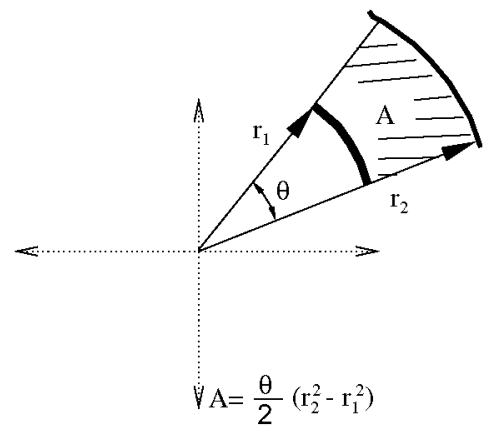

Fig. 1. Illustration of the general configuration used to derive $\mathbf{F}_{\mathrm{SF}}$. The hatched area represents the stacking fault ribbon enclosed between the two partials (thick segments). 
quantity, $\gamma \mathbf{n}$, to each dislocation segment such that it is now unequivocally defined by its Burgers vector $\mathbf{b}$, its local line tangent $\xi$ and its plane normal $\mathbf{n}$ weighted with the stacking fault energy $\gamma$.

Stacking faults are two-dimensional crystal defects that have a specific energy (per unit area). This means that, analogous to dislocation lines themselves, they cannot have an infinite extension and thus must end at the physical boundaries of the system (free surfaces, grain boundaries, etc.) or at a dislocation. Much like the conservation of the Burgers vector along a dislocation line is a requisite to satisfy the axiom of continuity, the vector $\gamma \mathbf{n}$ must be conserved along dislocation lines acting as physical limits of stacking fault surfaces. This conservation condition is most conveniently cast at the nodal level as $\sum_{i} \gamma \mathbf{n}_{i}=0$. In other words, as Fig. 2 shows, the value of $\gamma \mathbf{n}$ must be transferred along the dislocation line when a dissociation occurs. Naturally, for perfect dislocations resulting from complementary Shockley partials $\gamma \mathbf{n}=0$. This is consistent with the known fact that perfect dislocations are not physical boundaries of stacking fault surfaces.

Therefore, at any given dislocation node two rules must now be satisfied:

$$
\begin{aligned}
\sum_{i} \mathbf{b}_{i} & =0, \\
\sum_{i} \gamma \mathbf{n}_{i} & =0 .
\end{aligned}
$$

These rules provide a useful check for topological self-consistency during DD simulations involving multiple partial dislocations and suffice to describe all dislocation reactions and dissociations in fcc systems.

In practice, the most convenient initialization of a dislocation ensemble for plasticity simulations is to start with perfect dislocations $\left(\mathbf{b}=\frac{1}{2}\langle 110\rangle, \mathbf{n}=0\right)$, and let dissociations occur naturally according to Eqs. (3). Although attempts to model the force due to stacking faults have been published recently (Shenoy et al., 2000; Hardikar et al., 2001; Mohles, 2001; de Koning et al., 2003), we believe that our algorithm provides a level of generality not achieved in previous works.

\subsection{Topological changes relevant to partial dislocations}

\subsubsection{Cross-slip}

As noted earlier, in fec crystals cross-slip is a non-trivial process that involves dislocation reactions. Jackson (1984), Puschl (2002), and Caillard and Martin (2003), among others, have published comprehensive reviews covering the theoretical and experimental aspects of cross-slip in fcc metals. Among the many proposed mechanisms for cross-slip, two of them, namely the Friedel-Escaig (Escaig, 1968) and the Fleischer (Fleischer, 1959) models, stand out as the ones that have best withstood the test of experimental (Shoeck and Seeger, 1955; Bonneville and Escaig, 1979; Caillard and Martin, 1989; Bonneville et al., 1999) and atomistic (Duesbery, 1998; Rao et al., 1999; Rasmussen et al., 1997a, b; Vegge and Jacobsen, 2002; Mordehai et al., 2005; Pendurti et al., 2006) validation. In real crystals, both mechanisms can coexist simultaneously, and the relative likelihood of either one occurring is only determined by the intrinsic physical properties of the crystal and the external loading conditions. For consistency with the current experimental and theoretical

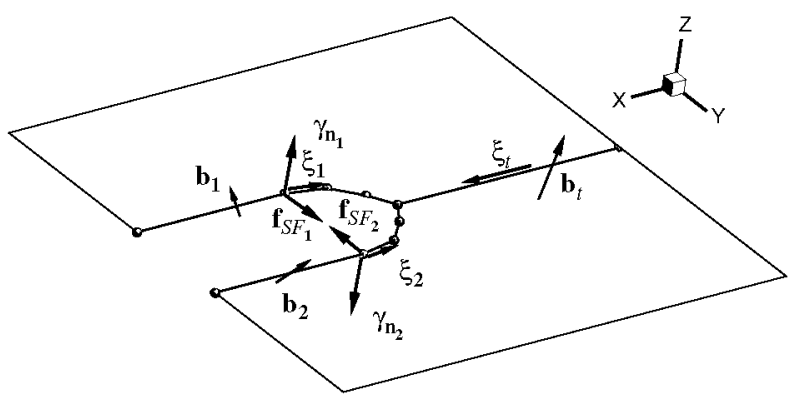

Fig. 2. Schematic diagram of the continuity rules applied to dislocation dissociation. The figure shows a perfect dislocation dissociating into two Shockley partials on a $\left\{\begin{array}{lll}1 & 1 & 1\end{array}\right\}$ plane. For the configuration shown: $\gamma \mathbf{n}_{1}=-\gamma \mathbf{n}_{2}, \gamma \mathbf{n}_{t}=0$. 
understanding regarding cross-slip processes, we have developed an algorithm compatible with the implementation of partial dislocations explained in Section 2.2 that includes both the Friedel-Escaig and the Fleischer mechanisms of close-packed cross-slip. A similar approach has been implemented by Wang et al. (2004) in their study of image effects in thin $\mathrm{Cu}$ films using parametric DD.

Therefore, our model allows dislocations to cross-slip by either mechanism, depending only upon the local nodal conditions, i.e. with no a priori assumption of what the cross-slip pathway should be. Both the Friedel-Escaig and the Fleischer mechanisms have been implemented as follows:

- Friedel-Escaig: At any given time, a node belonging to a perfect dislocation whose line tangent is locally parallel to the $\left\langle\begin{array}{lll}1 & 1 & 0\end{array}\right.$ direction of its Burgers vector may split on any $\left\{\begin{array}{lll}1 & 1 & 1\end{array}\right\}$ plane that contains it as long as it increases the rate of dissipated energy.

- Fleischer: At any given time, a node belonging to a Shockley partial dislocation may be split into an immobile stair-rod node at the point of splitting plus a Shockley node on the cross-slip plane.

These nodal splittings are consistent with Eq. (3) so that the new nodes are assigned the correct $\gamma \mathbf{n}$ and $\mathbf{b}$ vectors. The new configuration is then accepted or rejected on the basis of the power dissipation criterion.

It is worth stressing that the advantage of this procedure is that both of these mechanisms, together with the partial dislocation splitting explained in Section 2.2 operate simultaneously on every node at every time step and no assumptions are made as to which one should be favored. It is the applied stress, the configuration's geometry and the elastic parameters that determine, by way of the power dissipation criterion, which process should occur.

\subsubsection{Topological changes in multinodes}

The above splitting procedure works very efficiently for nodes with single connectivity, i.e. for nonbranching dislocation segments, but it is not sufficient to describe all possible dislocation configurations with more complex geometries. For nodes with multiple connectivity, the so-called physical nodes or junctions, a modified splitting algorithm must be used in order to allow for the topological changes associated with nodes where several dislocation segments converge. On top of the multinode splitting scheme existing in ParaDiS, which, in general, does not preserve the original node connectivity, we have developed a new algorithm to explore additional partial dislocation core transitions. Particularly, we are interested in a procedure that permits cross-slip of physical nodes.

This splitting algorithm must now ensure that the connectivity is maintained, and that the junction, as a physical entity, is not altered by any topological transformations. The optimum way to do this is to insert two nodes in two of the converging segments. How and where are these nodes inserted will depend on the specifics of the system under study. In our case, we must particularize this algorithm for fcc systems introducing appropriate geometric constraints consistent with dislocation theory. Specifically, if two of the segments arriving at a multinode, $S_{1}$ and $S_{2}$, are non-degenerate $\langle 110\rangle$ directions of the same $\left\{\begin{array}{lll}1 & 1 & 1\end{array}\right\}$ plane, defined by the product $\left(\xi_{1} \times \xi_{2}\right)$, then nodes are inserted if the following condition holds:

$$
\left(\mathbf{b}_{1} \times \xi_{1}\right) \neq\left(\xi_{1} \times \xi_{2}\right) \text { and }\left(\mathbf{b}_{2} \times \xi_{2}\right) \neq\left(\xi_{1} \times \xi_{2}\right) .
$$

The new nodes are inserted on each one of the involved segments at a distance $d_{a}$ from the original multinode (as for simple node splitting) and the connectivity of the newly inserted nodes is then increased by linking them to one another, thereby effectively 'bypassing' the original multinode. Next, b's and $\gamma \mathbf{n}$ 's are assigned to the new nodes taking into account their local environment so as to conform with the continuity rules (3). Finally, all possible configurations are compared against each other and the final choice (which may very well include the starting configuration) is made on the basis of our power dissipation criterion.

The procedure is schematically outlined in Fig. 3, where we have chosen a tetranode as example. Note that this mechanism acts in addition to - not instead of - the topological changes described in Sections 2.2 and 2.3.1. 
a

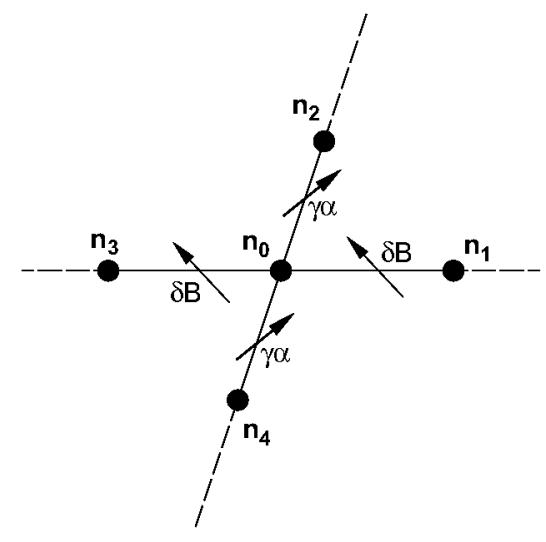

b

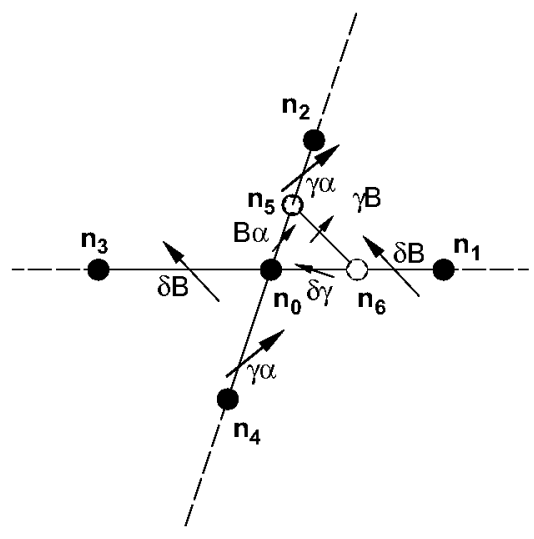

Fig. 3. (a) Schematic representation of a physical node (tetranode) resulting from the intersection of a $\delta \mathrm{B}$ Shockley partial and a $\gamma \alpha$ stairrod dislocation. (b) Splitting mechanism of the multinode, where two new nodes, $n_{5}$ and $n_{6}$, have been inserted and connected, and the connectivities and Burgers vectors of segments $\overline{n_{0} n_{1}}$ and $\overline{n_{0}} n_{2}$ have been updated to reflect the new topological state of the multinode. The Burgers vector directions and line tangents shown in the figures are not intended to define the character of the dislocations and are simply for labeling purposes.

\subsection{Model parametrization}

In order to parameterize our DD methodology, we have performed a series of MS simulations of perfect screw dislocation dissociation to measure the Shockley partial equilibrium separation. Subsequently, we have benchmarked our calibrated model against atomistic and analytical equilibrium spacing results at zero stress as a function of dislocation character. We have chosen $\mathrm{Cu}$ as our model $\mathrm{fcc}$ system, due to the extensive literature available regarding its plastic properties. We use material parameters derived from the interatomic potential used in the MS calculations, which corresponds to the Cu potential labeled 'EAM1' by Mishin et al. (2001). The values for the lattice parameter, the intrinsic stacking fault energy, and the shear modulus are, respectively, $a_{0}=3.615 \AA, \gamma=2.77 \times 10^{-3} \mathrm{eV} \AA^{-2}$, and after rotating the canonical shear modulus of $76.2 \mathrm{GPa}$ to the particular geometry used in the MS simulations (this is done to incorporate the lattice anisotropy in a simple way: see Appendix A), $\mu=63.2 \mathrm{GPa}$. For self-consistency with the elastic constants reported by Mishin et al. we have used a Poisson's ratio of 0.305 , which gives the correct value for the bulk modulus for the $\mu$ employed here.

\subsubsection{Shockley partial equilibrium separation distance}

For the MS calculations we use a Parrinello-Rahman, conjugate-gradient algorithm with convergent image summations (Cai et al., 2001). A perfect $\frac{1}{2} \quad$ screw dislocation was introduced in the MS computational box and allowed to relax for a given level of Escaig stress. The Escaig stress tensor is defined as that which produces a force only upon the edge components of the Shockley partials. Once the equilibrium configuration is attained, we calculate the separation distance by measuring the spacing between the dislocation cores of the resulting pair of partials. The cores have been identified by plotting the atomic disregistry along the edge and

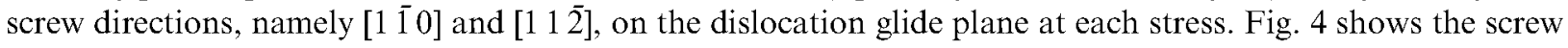
and edge components of the atomic disregistry at zero and $2000 \mathrm{MPa}$, where constriction is seen to occur. At zero stress, we measure a Shockley partial dislocation spacing of $2.0087 \mathrm{~nm}$, in good agreement with the $1.85 \mathrm{~nm}$ calculated from Escaig's model (Escaig, 1968), and in reasonable agreement with atomistic results of $1.52 \mathrm{~nm}$ by Duesbery (1998), and $1.77 \mathrm{~nm}$ by Henager and Hoagland (2005) using core fields. Other values obtained via analytical models show some discrepancy, however, such as the $0.97 \mathrm{~nm}$ calculated by Duesbery (1998) using anisotropic linear elasticity.

As the applied Escaig stress is increased, the partial dislocation spacing decreases in discrete steps commensurate with the interplanar distance down to full constriction. Fig. $4 \mathrm{~b}$ shows the atomic 


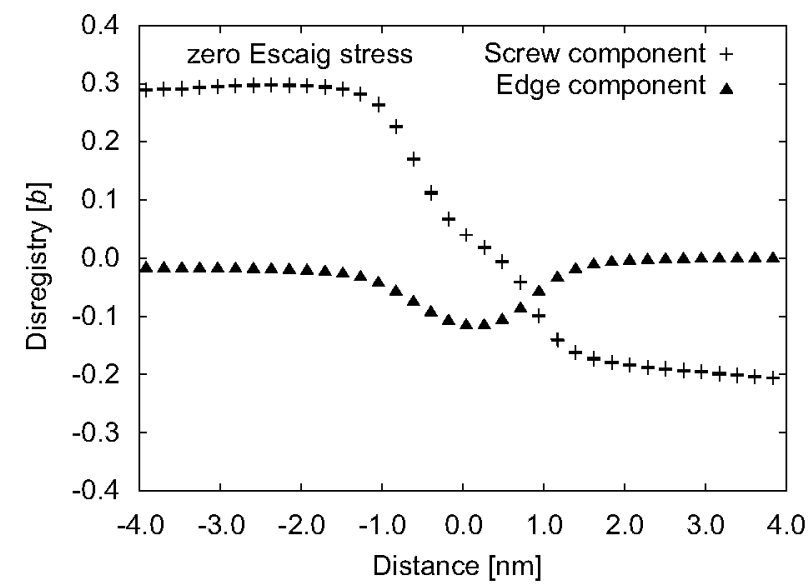

b

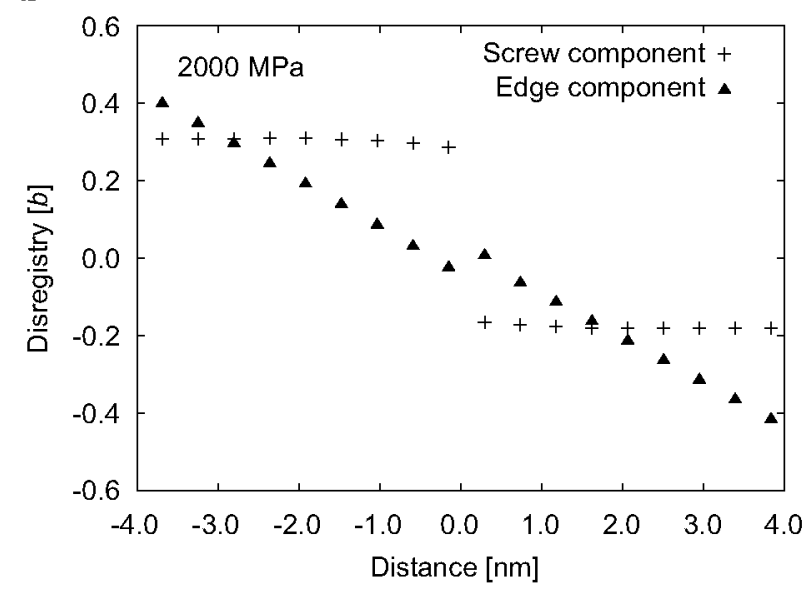

Fig. 4. Atomic disregistry in Burgers vector units on the dislocation glide plane for a perfect $\left.\frac{1}{2}[1] 0\right]$ screw dissociated into partials: (a) at zero stress we measure a distance of $2.0087 \mathrm{~nm}$, whereas at $2000 \mathrm{MPa}$ (b) full constriction has been attained.

disregistry for the dislocation configuration at $2000 \mathrm{MPa}$. The discontinuity in the screw component profile can be clearly appreciated, indicating the disappearance of most of the stacking fault ribbon. The edge component curve displays an offset close to the center of the box, which is a sign that, in reality, the dislocation is not fully constricted and some splitting is retained. Nevertheless, this is too small to produce an identifiable stacking fault, and, for all practical purposes, the dislocation can be considered fully constricted. The slope in the curve for the edge component simply represents the background homogeneous shear deformation resulting from the applied Escaig stress.

Following this procedure to measure separation distances, we have explored the constriction stress range from zero up to $2500 \mathrm{MPa}$. At stresses $\geqslant 2500 \mathrm{MPa}$, the dislocation is seen to spontaneously dissociate on a different $\left\{\begin{array}{lll}1 & 1 & 1\end{array}\right\}$ plane, i.e. akin to a Friedel-Escaig transition. The results are shown in Fig. 5. Clearly, between 2000 and $2500 \mathrm{MPa}$, the equilibrium separation distance lies on the same plateau, corresponding to one interplanar distance $(\sim 0.54 \mathrm{~nm})$. In other words, we take constriction to occur at $\sigma_{E}=2000 \mathrm{MPa}$ based both on the core disregistry criterion explained in the above paragraph, as well as on the fact that the minimum separation distance attained before spontaneous dissociation on a different $\left\{\begin{array}{lll}1 & 1 & 1\end{array}\right\}$ plane is first reached at that stress. We have also performed calculations applying a negative Escaig stress that tends to separate the partial dislocations. In DD, which lacks the energetics that ensures a finite stacking fault ribbon in between the two Shockleys, when the separation forces created by the applied stress surpass the value prescribed by $f_{\mathrm{SF}}$, we have unbound runaway partials. We have observed this to occur at approximately $-500 \mathrm{MPa}$. 


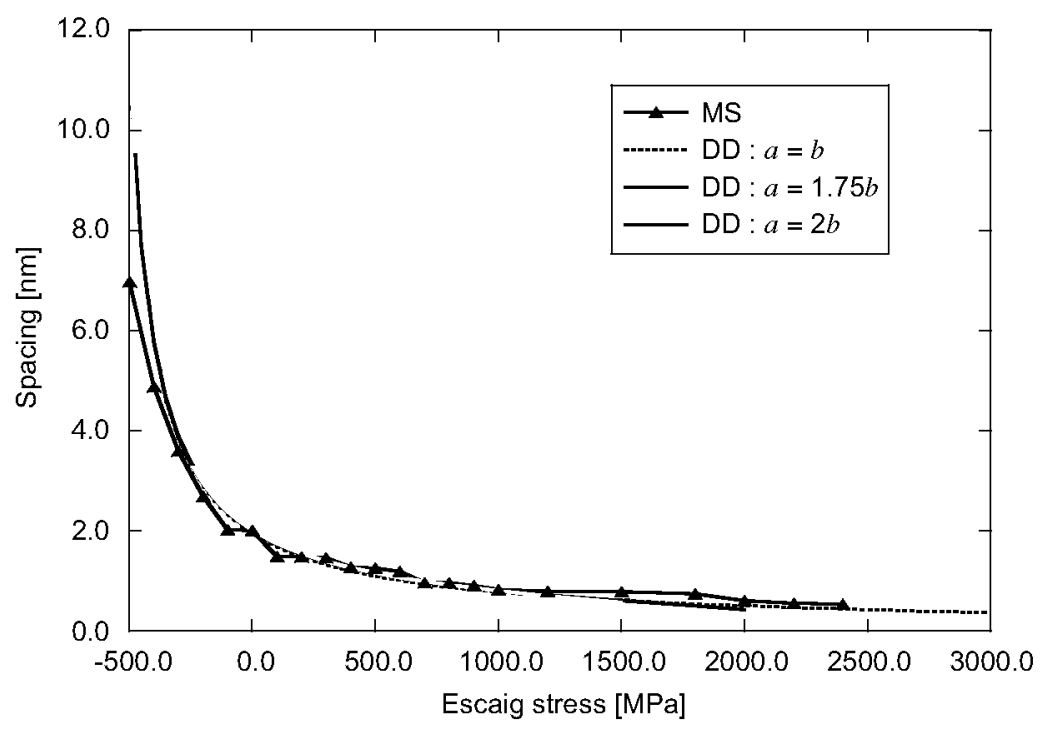

Fig. 5. Shockley partial separation distance as a function of Escaig stress for a screw dislocation. The MS curve has a descending staircase shape, a reflection of the quantized nature of the separation distance stemming from lattice discreteness. The best DD fit (obtained for $a=1.75 b$ ) is achieved by matching the MS results for the separation distance at zero stress and the stress at which constriction is attained. For this specific case, the constriction Escaig stress of $2000 \mathrm{MPa}$ is matched exactly.

We now possess all the information needed to calculate the value of $a$ that provides the best fit to the MS data. The observations that emanate from the MS calculations can be summarized as follows:

- We have established the stress range over which the dislocation is split into partials $\left(-500<\sigma_{E}<\right.$ $2500 \mathrm{MPa}$ ).

- The equilibrium (zero stress) separation distance is $2.0087 \mathrm{~nm}$.

- Constriction is taken to occur at $2000 \mathrm{MPa}$.

Fig. 5 shows three DD linear elastic curves for three different trial values of $a$. Clearly, the curve corresponding to $a=1.75 b$ (where $b$ is the modulus of the Burgers vector of the perfect dislocation) is found to provide the best fit to the MS data, and hereon we set $a=1.75 b=0.447 \mathrm{~nm}$ for all subsequent calculations. Though the agreement between both approaches is remarkable, the comparison between the two curves is burdened by limitations coming from both sides. For example, the assumption of isotropy in DD ignores the crystal orientation of the relevant slip systems in fcc metals. For its part, despite using methods for minimizing image interactions, the use of periodic boundaries in MS causes artifacts that may affect the equilibrium configurations of the simulated structures.

\subsubsection{Friction coefficients and core energies}

Next, we turn to the calculation of the friction coefficients attendant to the mobility functions. In the present DD implementation, once all the forces acting upon a given segment are known, its velocity can be determined using the following kinetic law:

$$
\begin{aligned}
& \mathbf{f}=B \mathbf{v}, \\
& B=B_{\mathrm{g}}(\mathbf{m} \otimes \mathbf{m})+B_{\mathrm{c}}(\mathbf{n} \otimes \mathbf{n})+B_{1}(\xi \otimes \xi)
\end{aligned}
$$

where $B$ is the drag coefficient tensor, $B_{\mathrm{g}}$ and $B_{\mathrm{c}}$ are temperature-dependent friction coefficients for motion along the glide and climb directions, and $B_{1}$ is the drag coefficient associated with a node in response to the configurational forces responsible for the equilibrium line shape acting on it (Arsenlis et al., 2007). Here, $\mathbf{n}$ and $\mathbf{m}$ are unit vectors defined as $\mathbf{n} \equiv(\mathbf{b} \times \xi) /\|\mathbf{b} \times \xi\|^{2}$ and $\mathbf{m} \equiv \mathbf{n} \times \xi$. This expression for $B$ guarantees its 
Table 1

Partial dislocation core energies for core widths, $a$ of $1.75 b$ and $b_{\mathrm{p}}$ as calculated with MS simulations for $\mathrm{Cu}$

\begin{tabular}{llllr}
\hline Dislocation type & $\mathbf{b}_{\mathbf{p}}$ & $b_{\mathrm{p}}$ & $\varepsilon(1.75 b)$ & $\varepsilon\left(b_{\mathrm{p}}\right)$ \\
\hline Shockley partial & $\frac{1}{6}(112)$ & 1.476 & 0.317 & 0.189 \\
Stair-rod & $\frac{1}{6}(110)$ & 0.852 & 0.193 & 0.062 \\
Hirth partial & $\frac{1}{3}(001)$ & 1.205 & 1.451 & 1.085 \\
Frank partial & $\frac{1}{3}(11)$ & 2.088 & 1.835 & 1.450 \\
Perfect dislocation & $\frac{1}{2}(110)$ & 2.557 & 0.530 & 0.236 \\
\hline
\end{tabular}

Burgers vector moduli are given in $\AA$, while core energies are given in units of $\mathrm{eV}^{-1}$. The core configurations were obtained following the procedure of $\mathrm{Li}$ et al. (2004) with the modification for the perfect dislocation outlined in Bulatov et al. (1995).

invertibility so that the mobility tensor $M=B^{-1}$ can be applied to obtain the velocities as $\mathbf{v}=M \mathbf{f}$. For this work, the critical coefficient is $B_{\mathrm{g}} \gg B_{\mathrm{c}}, B_{1}$, which is dislocation character dependent and constant in the lowvelocity regime. A simple model for $B_{\mathrm{g}}$ takes the form:

$$
B_{\mathrm{g}}=B_{\mathrm{s}} \cos ^{2} \theta+B_{\mathrm{e}} \sin ^{2} \theta
$$

where $\theta$ is the angle between the dislocation line and the Burgers vector and $B_{\mathrm{s}}$ and $B_{\mathrm{e}}$ are the friction coefficients for the perfect screw and edge dislocations, respectively. Following the procedure described by Marian and Caro (2006), we have carried out large scale three-dimensional molecular dynamics simulations to calculate the values of the respective friction coefficients. For this potential, we have obtained $B_{\mathrm{s}}=$ $9.82 \times 10^{-6} \mathrm{Pas}$ and $B_{\mathrm{e}}=2.31 \times 10^{-6} \mathrm{~Pa}$ s, respectively.

The final ingredient extracted from MS calculations to be used in our DD simulations is the dislocation core (inelastic) energy for a fixed core width of $a=1.75 b$ for all possible partial dislocations considered. In our model, the force on node $i$ associated with the spatial derivatives of the core energy, $\varepsilon$, of the system is $\mathbf{F}_{i}^{\mathrm{c}}=\sum_{j} \mathbf{f}_{i j}^{\mathrm{c}}$, where $\mathbf{f}_{i j}^{\mathrm{c}}$ is simply taken as (Arsenlis et al., 2007):

$$
\mathbf{f}_{i j}^{\mathrm{c}}=\varepsilon\left(\mathbf{b}_{i j}\right) \xi_{i j} \text {. }
$$

In other words, the force $\mathbf{f}_{i j}^{\mathrm{c}}$ can be considered as the part of the core force on node $i$ attributable to its connection to node $j$. We use the procedure outlined by Li et al. (2004), coupled with the non-singular elastic formulation described in Section 2.1.1, to calculate the elastic energy of a dislocation dipole and subtract the value of $\varepsilon$. Table 1 shows all the relevant core energies for both $a=1.75 b$ and, for academic interest, $a=b_{\mathrm{p}}$, where $b_{\mathrm{p}}$ is the Burgers vector of the corresponding partial dislocation. The partition of the total MS energy between elastic and core energies has been done on the basis of a parameter, $a$, which is of course non-unique. As well, in general, each dislocation type need not have the same $a$ as considered here. What this means is that the set of core energies given here is chosen to fit certain properties of our choosing and that the numbers given in Table 1 are as good a representation of core energies as any other.

\section{Results}

As discussed above, our methodology includes a panoply of topological transformations for each dislocation node that reflects the special character of the fcc lattice. At any given instant, depending on the local geometry and stress conditions, topology changes are accepted or rejected on the basis of the maximum power dissipation criterion explained in Section 2.2. This criterion determines which transformations will occur but it does not provide precise quantitative information, such as partial dislocation separation distance, critical stresses for cross-slip or strength of junctions. Below, we perform quantitative predictions relevant to fcc metals with the fully parameterized method. Specifically, we will focus on the calculation of the strength of dislocation junctions, the formation of SFT and the mapping of the cross-slip space for a single screw dislocation. Our goal is to show that, after fitting to simple (MS) systems, our parameterized DD model effectively captures the behavior of more complex systems. 


\subsection{Simple validation test: calculation of the separation distance as a function of dislocation character}

We start by performing a simple validation test, namely the calculation of the equilibrium spacing as a function of stress and dislocation character. Our intention is to benchmark our results against existing atomistic data at zero stress, and to calculate the critical constriction stress in each case. For the latter calculation, the critical stress is taken to be that at which constriction is attained, i.e. when the non-singular stress barrier is surpassed. Results are shown in Fig. 6, where we have considered perfect screw and edge dislocations as well as $30^{\circ}$ and $60^{\circ}$ mixed dislocations. We can see that the agreement of our curves with the available atomistic data (from Henager and Hoagland, 2005) at zero Escaig stress is quite reasonable. The critical constriction stresses for the perfect screw, $30^{\circ}, 60^{\circ}$ and perfect edge dislocations are, respectively, 2000 , 1900,1700 and $1500 \mathrm{MPa}$. Approximately, the critical constriction stress varies linearly with the dislocation character.

\subsection{Strength of dislocation locks}

In the absence of significant lattice resistance, the main impediment to dislocation glide in pure fcc materials is forest interactions, i.e. intersections of mobile dislocations with dislocations lying on other slip systems. There are a large number of potentially important intersections mechanisms but here we focus on the reactions between attractive extended dislocations that form strong barriers to the motion of other dislocations. The strength of these barriers is important to estimate the strength coefficient matrix, $\alpha_{i j}$ in Taylor's equation for hardening:

$$
\tau_{i j}=\mu b \sqrt{\alpha_{i j} \rho_{j}}
$$

where $\tau_{i j}$ is the critical resolved shear strength (CRSS) in slip system $i, \mu$ and $b$, as above, are the shear modulus and the Burgers vector's magnitude and $\rho_{j}$ is the dislocation density in slip system $j$. Eq. (7) relates the strength in slip system $i$ due to dislocations in slip system $j$. To obtain the total CRSS in slip system $i$, one simply adds all the different contributions from all the other slip systems. By virtue of symmetry, the number of independent $\alpha_{i j}$ among the 12 mutually interacting slip systems in fec crystals reduces to only six. However, two of them involve weak elastic interactions between dislocations gliding on parallel slip planes (Basinski and Basinski, 1979; Madec et al., 2003), and here we only consider those interactions that result in strong locks, namely the Lomer-Cottrell locks, the glissile junctions, co-linear junctions and Hirth locks (Hirth, 1961; Franciosi et al., 1980).

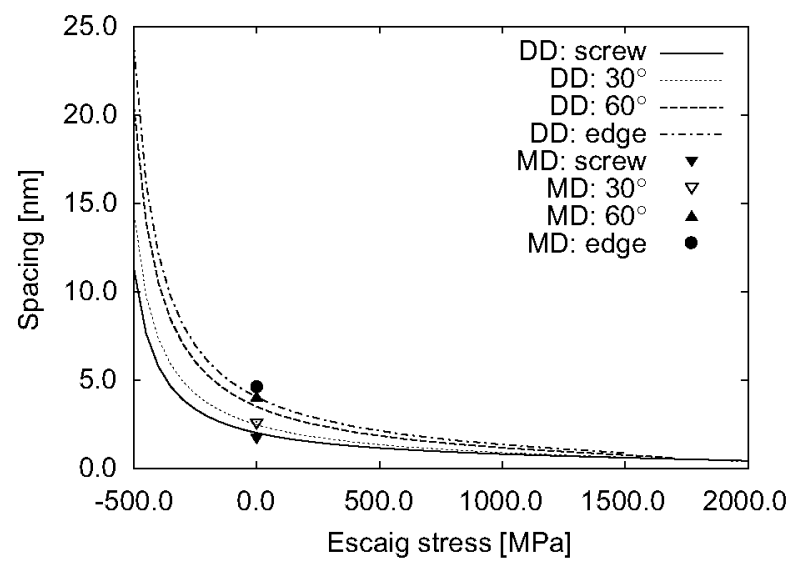

Fig. 6. Equilibrium separation distance between Shockley partials as a function of constricting Escaig stress for a pure screw (already shown in Fig. 5), $30^{\circ}$ and $60^{\circ}$ mixed dislocations, and a perfect edge dislocation. All curves have been obtained with the model as parameterized in Section 2.4. Our curves are compared with the data points obtained by Henager and Hoagland (2005) using atomistic simulations. 
There have been several works on dislocation junctions in fec metals both using DD and atomistic simulations. For example, Zhou et al. (1998) investigated the formation of perpendicular extended dislocations using large-scale molecular dynamics simulations and found it to be a complex process involving zipping, bowing and unzipping, leading to the formation of a unit jog. On the other hand, Shenoy et al. (2000) and Rodney and Phillips (1999) have studied the strength of the Lomer-Cottrell junction in $\mathrm{Al}$ using more coarsened methods (DD and the quasicontinuum method, respectively) and found relatively good agreement with atomistic and continuum calculations. These and other works (Shin et al., 2001; Dupuy and Fivel, 2002; Madec et al., 2002; Kubin et al., 2003; Depres et al., 2003; Shehadeh et al., 2005) have mostly focused on the Lomer-Cottrell lock, as this has traditionally been thought of as the strongest of all attractive junctions. Recently, Madec et al. (2003) have proposed that the junction governing the hardening in fcc crystals is that resulting from interactions between dislocations with co-linear Burgers vectors gliding on intersecting planes. Their argument is sustained on the fact that, based on the computed magnitude of the hardening coefficient $\alpha$, co-linear interactions contribute the most to the total hardening in Eq. (7). Devincre et al. (2006) have further refined and confirmed Madec's calculations in a recent publication.

Here we study each attractive reaction and we investigate the minimum stress required to break them once they are formed spontaneously. In principle, all the details of each dislocation reaction are contained in the corresponding yield curve as a function of the interaction angle (for example, Shenoy et al., 2000; Shin et al., 2001; Dupuy and Fivel, 2002). Strength yield curves are typically obtained as the loci of the pairs of primary, $\tau_{1}$, and forest, $\tau_{2}$, stresses that produce junction dissolution. We are intent on exploring these fcc junctions with our newly developed methodology in an attempt to establish whether core energetics - via the line stiffness model - and partial dislocation reactions provide any additional insights beyond the classical, purely elastic treatment utilized to date in the literature. To this end, we have only computed the three points that define the positive quadrant of the yield curve -i.e. $\left(\tau_{1}, \tau_{2}=0\right),\left(\tau_{1}=0, \tau_{2}\right)$ and $\left(\tau_{1}=\tau_{2}\right)$, our main aim simply being to demonstrate the numerical capabilities of our method. Except where noted, all reactions have been simulated for parallel dislocation segments with $205-\mathrm{nm}$ long segments. The equilibrium configurations for each reaction are shown schematically in Fig. 7.

Lomer-Cottrell

Hirth

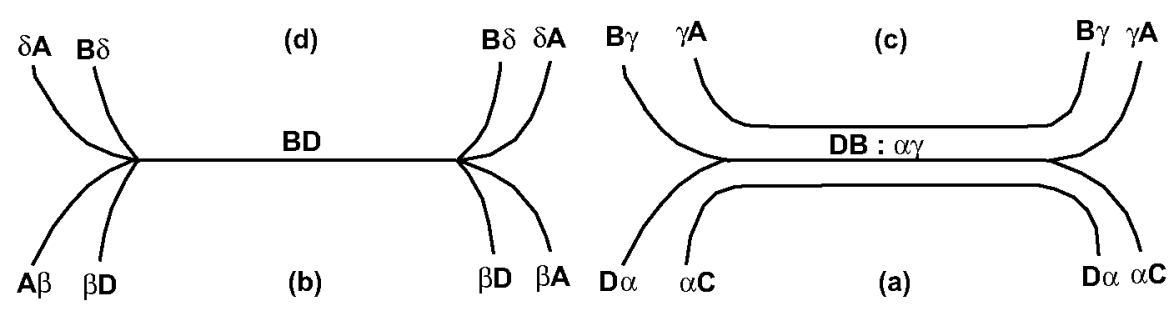

co-planar

co-linear
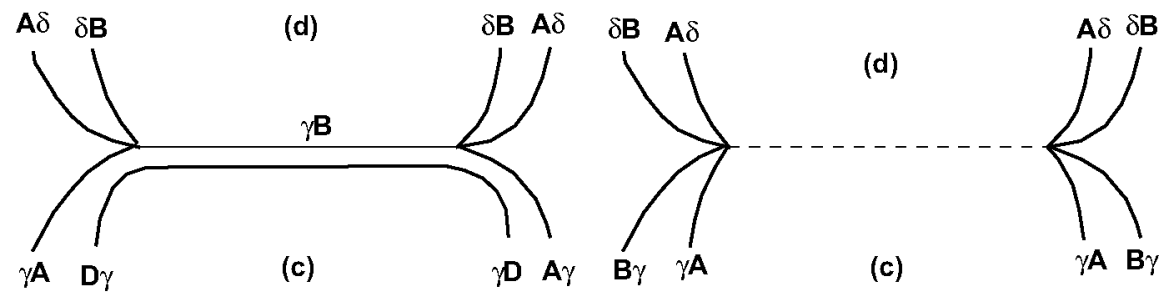

Fig. 7. Schematic representation of each junction geometry in equilibrium as obtained with our methodology. With the notable exception of the co-planar lock, all junctions are symmetric with respect to the intersection line of the glide and forest planes (for the parallel initial configuration treated here). Thompson's notation is used for clarity. 


\subsubsection{Lomer-Cottrell junction}

The Lomer-Cottrell lock appears when two perfect dislocations of the same $\left\{\begin{array}{lll}1 & 1 & 1\end{array}\right\}$ zone occur gliding on different planes. The two leading partials are attracted to one another and react along the $\langle 110\rangle$ line of intersection between the two planes to form a pure-edge $\frac{1}{6}\left\langle\begin{array}{ll}1 & 10\rangle\end{array}\right)$ stair-rod partial dislocation. Both the elastic self-energy and the core energy of a stair-rod dislocation are relatively low (see Table 1) which makes this reaction very stable and the lock quite strong. Here, we have studied Lomer-Cottrell junctions with geometries involving a pair of perfect dislocations with $\mathbf{b}_{1}=\frac{1}{2}\left[\begin{array}{lll}1 & 1 & 0\end{array}\right]$ and $\mathbf{b}_{2}=\frac{1}{2} \quad$ gliding, respectively, on

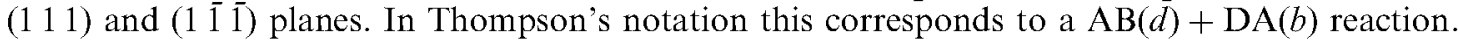

For the geometry considered, with relatively long line segments $(>200 \mathrm{~nm})$ lying parallel to one another, the two trailing partials collapse onto the stair-rod dislocation to form a perfect $\frac{1}{2}\langle 110\rangle$ Lomer dislocation, capable of gliding on $\{001\}$-type planes (see Fig. 7). This is a real effect, since when we fix $\gamma=0$ in our simulations, this collapse does not occur. Indeed, we have simulated an infinite Lomer dislocation using MS and it is stable. Nevertheless, as we shall see below, this phenomenon is quite sensitive to the initial reaction geometry.

Another important aspect to consider is the two types of configurations that are geometrically possible, namely the case where the angle between the two intersecting planes is acute and the case when it is obtuse. The formation of an obtuse Lomer-Cottrell junction entails having extrinsic stacking faults (Hirth and Lothe's, 1992; Sun et al., 1991; Bonneville and Vanderschaeve, 1998), which are considerably higher in energy than intrinsic ones, such as those found for an acute lock ( $84.5 \mathrm{vs} .44 .4 \mathrm{~mJ} \mathrm{~m}^{-2}$, according to our interatomic potential). The main implication of this is that obtuse configurations can be considered practically forbidden for our purposes, a fact supported by our own atomistic simulations of junction formation and cross-slip. The outcome is precisely that, although geometrically plausible, the energy cost incurred when creating an extrinsic fault in our $\mathrm{Cu}$ model is far too high for these transitions to be allowed. This observation is supported by Bonneville and Vanderschaeve's (1998) linear elastic analysis of Lomer-Cottrell junctions reconciled with experimental observations.

Having established these constraints, we now turn to the calculation of the lock strength. As the geometry in Fig. 7 suggests, in principle, the yield curve of the Lomer-Cottrell is symmetric with respect to stresses applied on the forest and glide planes and thus $\left.\sigma_{1}\right|_{\sigma_{2}=0}=\left.\sigma_{2}\right|_{\sigma_{1}=0}$. For this particular geometry, the stress required to dissolve the junction under this stress state is rather large, $\sim 2850 \mathrm{MPa}(0.0452 \mu)$, whereas for the case of equally stressed planes we obtain $\sigma_{1}=\sigma_{2}=712.5$, or $0.0113 \mu$. The normalized strength for the $\left(\sigma_{1}=\sigma_{2}\right)$ stress point, along with the slip geometry and the corresponding $\mathbf{b}$ and $\gamma \mathbf{n}$ reactions are shown in Table 2.

Another useful check to validate our methodology is to compare with the particular geometry chosen in several studies (Rodney and Phillips, 1999; Shenoy et al., 2000; Shin et al., 2001), where the initial dislocation lines form $60^{\circ}$ with the line of intersection of their respective glide planes. Fig. 8 shows the final structure after relaxation, in good qualitative agreement with mentioned studies. For this $60^{\circ}$ configuration we obtained a junction length of $7.7 \mathrm{~nm}$, while, for example, Shin et al. (2001) obtained values in the range of $6.6-9.0 \mathrm{~nm}$ using a variety of methods. However, our calculated junction strength is much weaker, of the order of $0.0015 \mu$, which may be related to a material dependence. As the figure shows, in this case, the collapse onto a Lomer dislocation does not occur, in agreement with the quasicontinuum results by Rodney and Phillips (1999), and the elastic analyses by Shenoy et al. (2000) and Shin et al. (2001) using dislocation partials.

\subsubsection{Hirth lock}

The Hirth lock is formed when two perfect dislocations with perpendicular Burgers vectors glide on intersecting planes. This reaction results in a sessile $\frac{1}{3}\langle 001\rangle$ Hirth partial dislocation flanked by the two trailing partials. The Hirth lock is the conjugate of the Lomer-Cottrell in that only the obtuse configurations will produce an intrinsic fault. Thus, in this case we need not consider the acute configuration. For these simulations we have also chosen two perfect dislocations with slip systems $\frac{1}{2} \quad\left(\begin{array}{lll}\overline{1} & \overline{1} & 1) \text { and } \frac{1}{2}\end{array} \quad(\overline{1} 1 \overline{1})(\mathrm{CD}(a)+\mathrm{BA}(c)\right.$ in Thompson's notation). The normalized junction strengths are given in Table 2 for the three points in the positive quadrant of the yield curve. In the same fashion as the Lomer-Cottrell, the Hirth lock is symmetric about the bisectrix plane that contains the intersection line of the forest and the glide planes (see Fig. 7). Thus, the strength of the junction is invariant with respect to which is the activated plane and equal to $201 \mathrm{MPa}$ or $0.0032 \mu$. On the other hand, the strength for $\left(\sigma_{1}=\sigma_{2}\right)$ is $208 \mathrm{MPa}$ or $0.0033 \mu$. The normalized strength for the $\left(\sigma_{1}, 0\right)$ stress point along with the slip geometry and the corresponding $\mathbf{b}$ and $\gamma \mathbf{n}$ reactions are shown in Table 2. 
Table 2

Geometry and strength of the Lomer-Cottrell, Hirth and co-planar junctions

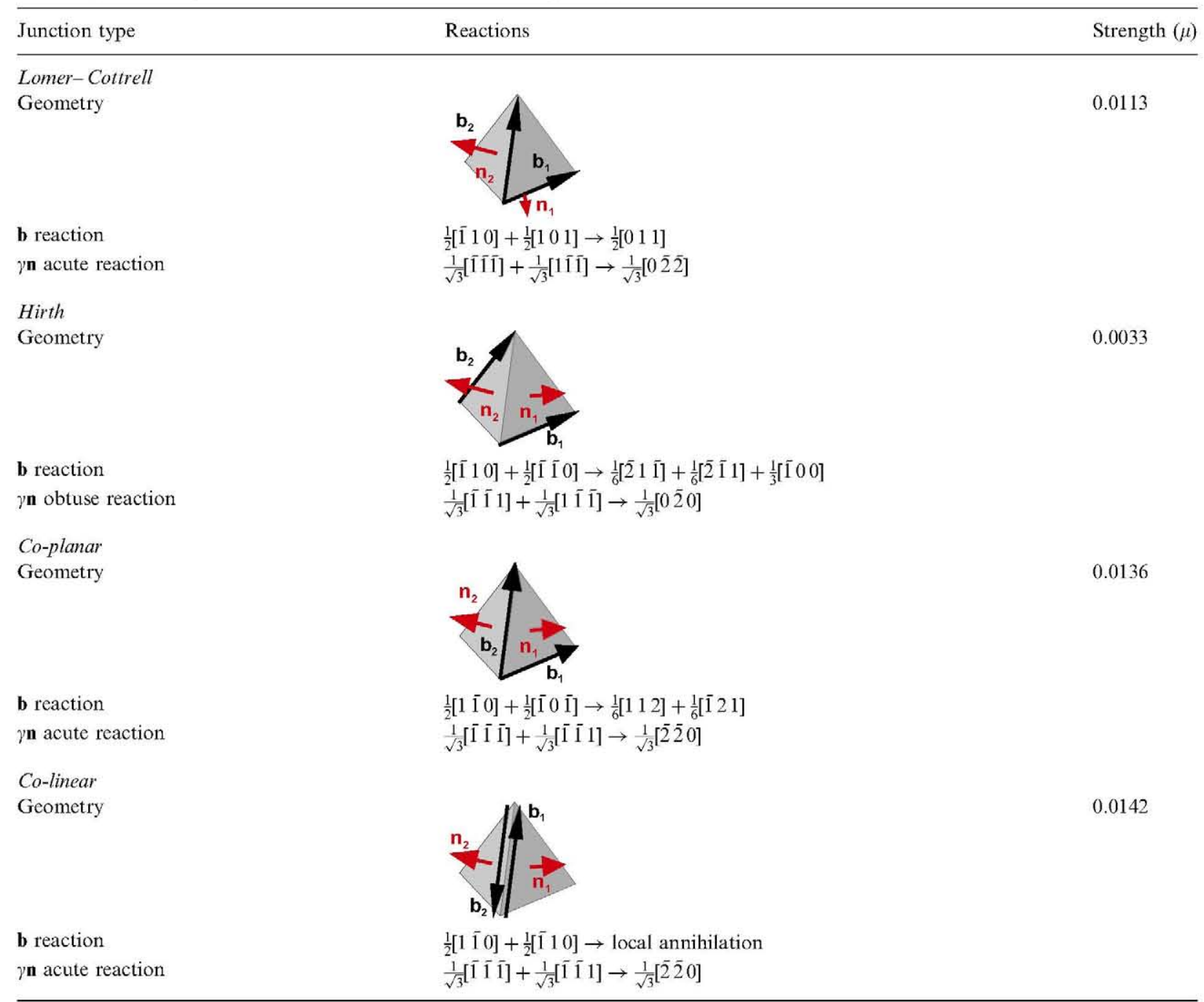

The relative geometries are given in terms of the Thompson tetrahedron. The values for the strength correspond to the $\left(\sigma_{1}=\sigma_{2}\right)$ points of the yield curve (both the glide and the forest planes equally activated). For the Lomer-Cottrell, Hirth and co-linear junctions. For the coplanar junction only the $\left(\sigma_{1}, 0\right)$ point is given, as there is no stable $\left(\sigma_{1}=\sigma_{2}\right)$ state in this case (see text). The b and $\gamma \mathbf{n}$ reactions are also shown for reference.

Based on purely elastic considerations, Hirth (1961) considered this reaction to be the strongest barrier to dislocation glide in fcc metals. Contrarily, our calculations show that the strength of this junction is lowest among all four considered, for the parallel geometry and zipping length of $\sim 205 \mathrm{~nm}$. From the point of view of the reacting geometry, Kubin et al. (2003) have recently extended Hirth's analysis by mapping the dislocation reaction space as a function of dislocation character. They find that only a very small deviation from perfectly parallel reactant segments is allowed for the formation of a Hirth lock. Nevertheless, they also found the Hirth lock to be the weakest among all fcc junctions.

\subsubsection{Co-planar junction}

A co-planar junction - also referred to as 'glissile' junction, is formed when two perfect dislocations lying on different planes react along the plane intersection to produce the third perfect dislocation of the $\{111\}$ zone whose glide plane normal is $\mathbf{b}_{1} \times \mathbf{b}_{2}$. The simulated geometry is similar to the Lomer-Cottrell, except that in 


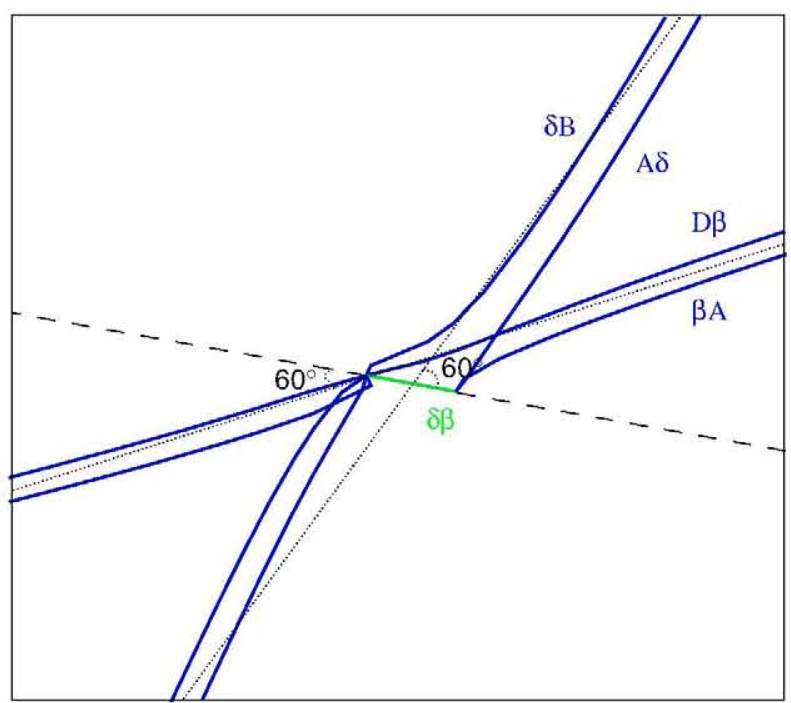

Fig. 8. Equilibrium structure of the Lomer-Cottrell lock for the $60^{\circ}$ geometry as obtained with our model. The dashed line represents the intersection between the two glide planes, whereas the dotted lines give the initial orientation of the reacting dislocations. The length of the stair-rod segment (in green) is $7.7 \mathrm{~nm}$.

this case one of the reacting dislocations, the $\frac{1}{2}\left[\begin{array}{ll}1 & 1\end{array}\right]$, is a perfect screw dislocation on the (1 111$)$ plane,

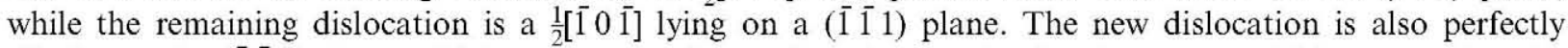
glissile on the (i $\overline{1} 1)$ plane, which acts as the forest plane in our simulated geometry. This means that the only stress component governing junction dissolution in this case is $\sigma_{1}$, as $\sigma_{2}$ simply acts as a glide component on the forest plane. Thus, only those stress combinations for which the applied $\sigma_{1}$ can overcome the glide force created by $\sigma_{2}$ are relevant for junction strength calculations. Presumably, this will result in a discontinuous yield curve with a gap at low $\sigma_{1}$ stresses. Indeed, we found that there is neither $\left(0, \sigma_{2}\right)$ nor $\left(\sigma_{1}=\sigma_{2}\right)$ solution for this lock, as the new dislocation simply glides on the forest plane and the junction nodes act as a Frank-Read source. For the $\left(\sigma_{1}, 0\right)$ we obtain a value of $656 \mathrm{MPa}(0.0136 \mu)$ to recover the first Shockley partial. This value along with the slip geometry and the corresponding $\mathbf{b}$ and $\gamma \mathbf{n}$ reactions are given in Table 2 .

Because of its glissile nature on the forest plane, the co-planar junction is the only non-symmetric one among the four fcc locks studied. The schematic equilibrium configuration is shown in Fig. 7, where the $(d)$ plane is the initial glide plane and $(c)$ is the forest plane.

\subsubsection{Co-linear junction}

The co-linear junction results from the mutual annihilation of locally screw segments of perfect dislocations with opposite Burgers vectors gliding on different $\left\{\begin{array}{llll}1 & 1 & 1\end{array}\right\}$ planes. These dislocations attract one another and, when sufficiently close, may alter the local character of the dislocation line affecting the reaction length. The geometry we have used in this case involves a $\mathrm{BA}(d)$ and a $\mathrm{AB}(c)$ dislocations annihilating along their glide plane intersection (see Table 2 for the numeric expressions). By way of this reaction, segments of both dislocations become connected on either sides of the annihilated length. If one takes into account the extended nature of dislocations in the fcc lattice, the reacting dislocations form tetranodes that are sessile on either of the two original planes (although they can glide along the line). Therefore, these nodes effectively act as pinning points, contributing to hardening. The dissolution of this lock involves the unzipping of the virtual junction by glide of the tetranodes along the intersection line. This is precisely the dissolution mechanism for the point of the yield curve with equally activated planes, for which $\sigma_{1}=\sigma_{2}=898.3 \mathrm{MPa}$ or $0.0142 \mu$ (see Table 2 for more details). The behavior is somewhat different when only one of the planes is activated. In those cases, the tetranodes act partially as dislocation sources via their activated dislocation segments, which increases the amount of stress required to break the lock: $\sim 1740 \mathrm{MPa}$ or $0.0275 \mu$. 


\subsection{Formation of SFT}

We now turn to another important aspect of fcc metals, namely the treatment of Frank partial dislocations and the formation of SFT. The Frank partial is an edge dislocation and since its Burgers vector is not contained in a close-packed $\left\{\begin{array}{ll}1 & 1\end{array}\right\}$ plane it cannot glide conservatively under the action of an applied stress. Moreover, the Burgers vector of a Frank partial is not a lattice vector, and therefore, a Frank loop encloses a stacking fault. If the stacking fault energy is sufficiently high, the Frank loop will be stable, but in low stacking-fault energy metals the loop must unfault to become stable. Depending on size, a Frank loop can unfault by one of the two mechanisms, namely by a Shockley partial sweeping the habit plane leaving a perfect dislocation loop in its wake, or by dissociation into a low-energy stair-rod dislocation and a Shockley partial on an intersecting $\left\{\begin{array}{llll}1 & 1 & 1\end{array}\right\}$ plane. As we show below, the latter mechanism is responsible for the formation of SFTs. Both of these mechanisms are allowed in our simulations, and again, which one should operate is solely determined on the basis of the power dissipation criterion.

Due to its higher complexity and interest, here we focus on the formation of a SFT from a Frank loop in $\mathrm{Cu}$. We start from a triangular 5.8-nm loop with sides aligned with $\langle 110\rangle$ directions. We then let the system relax at zero stress by allowing the mechanisms explained in the previous paragraph and applying rules (3). A sequence of the simulation is shown in Fig. 9. Each side of the original triangle is a Frank segment with Burgers vector $\frac{1}{3}\left[\begin{array}{lll}1 & 1 & 1\end{array}\right]$ (shown in black). Each segment undergoes a dissociation of the type:

$$
\frac{1}{3}\left[\begin{array}{lll}
1 & 1 & 1
\end{array}\right] \rightarrow \frac{1}{6}\left[\begin{array}{lll}
1 & 1 & 0
\end{array}\right]+\frac{1}{6}\left[\begin{array}{lll}
1 & 1 & 2
\end{array}\right]
$$
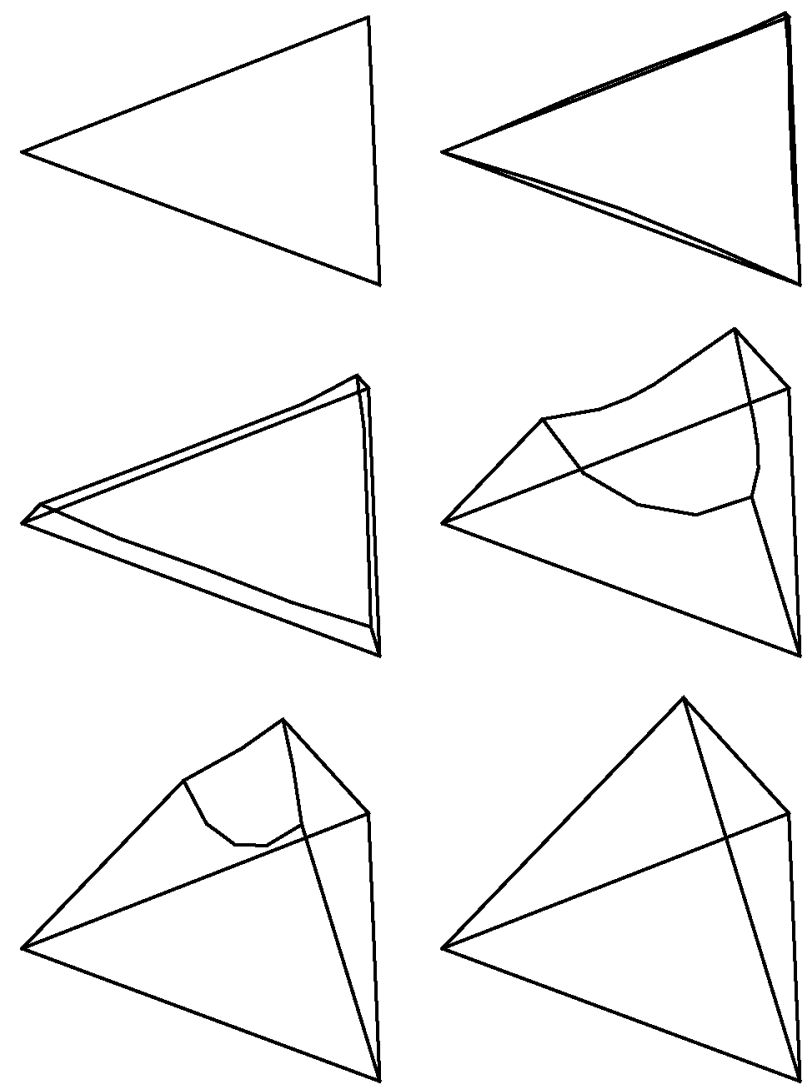

Fig. 9. Simulation of the transformation of a 4-nm triangular Frank loop into a perfect stacking fault tetrahedron. Dislocation segments are color-coded according to their Burgers vector: in black are Frank partials, blue are Shockley partials and green segments are stair-rod dislocations. 
into a Shockley partial and a stair-rod dislocation. Basically, the Shockley partials are each glissile on the remaining $\left\{\begin{array}{llll}1 & 1 & 1\end{array}\right\}$ planes of the Thompson tetrahedron and react with one another on the edges of it. Although dissociation initially occurs close to the center of the triangle sides, as we can see, in our case the Shockley partials are more inclined to react among themselves than to glide along the facets of the tetrahedron, reason why reactions at the edges occur in advance of glide on the $\{111\}$ planes. This means that the edges grow faster than the facets, something commonly assumed to occur inversely. To ensure that this is not an artifact of the simulation introduced by the line tension effects we look at the node insertion criterion. The initial triangle contains only three nodes; then, as the dissociation proceeds, more nodes are introduced automatically to maintain the equilibrium shape of the new partials. Our criterion for the insertion of nodes can be fine-tuned to achieve a satisfactory line shape but we have seen little influence of this on the final dynamics. In other words, this effect appears to be a consequence of the system dynamics, governed by the stacking fault energy and the power dissipation criterion, and not an artifact resulting from discrete effects. In fact, these observation has been confirmed recently by Lee et al. (2007) using molecular dynamics simulations.

The initial dissociation of the Frank loop as written in Eq. (8) is governed by Eqs. (3), whereby new Burgers and $\gamma \mathbf{n}$ vectors are assigned to the product dislocations in Eq. (8). Otherwise, the process is solely driven by the power dissipation criterion explained earlier. As a useful application, we have computed at $\approx 22 \mathrm{~nm}$ the critical size above which the dissociation of a triangular Frank loop no longer results in the formation of a full SFT. Instead, we observe the formation of a truncated tetrahedron with three Shockley partials bounding the truncated portion. The relatively large stacking fault area prevents the closure of these partials into stair rods to form the full structure. The first SFT size at which we see truncation is $L=22.42 \mathrm{~nm}$, with a ratio $L^{\prime} / L=0.58$, where $L^{\prime}$ is the truncated edge's size. A direct comparison with Hirth and Lothe's (1992) isotropic linear elastic analysis using the material models in this paper for this $L^{\prime} / L$ ratio yielded a value of $L \sim 15.0 \mathrm{~nm}$.

\subsection{Cross-slip}

We now turn to the application of our methodology to cross-slip. Our goal is to map the stress space for which cross-slip occurs at $0 \mathrm{~K}$ using the mechanisms outlined in Section 2.3.1. Fig. 10 shows the geometry of the cross-slip space of interest. In essence, we are interested only in the four components of the stress tensor that are relevant for cross-slip, namely Escaig $(E)$ and glide $(G)$ stress applied independently on the primary (glide) and secondary (cross-slip) planes (Duesbery et al., 1992b):

$$
\begin{aligned}
& \sigma_{E 1}=\tilde{\sigma}: \mathbf{S}_{E 1}, \\
& \sigma_{G 1}=\tilde{\sigma}: \mathbf{S}_{G 1}, \\
& \sigma_{E 2}=\tilde{\sigma}: \mathbf{S}_{E 2}, \\
& \sigma_{G 2}=\tilde{\sigma}: \mathbf{S}_{G 2},
\end{aligned}
$$

where $\sigma_{\alpha i}$ are the resolved stresses of each type $(\alpha=E, G)$ on each plane $(i=1,2), \tilde{\sigma}$ is the local stress tensor, and $\mathbf{S}_{\alpha i}$ are projection matrices.

The stress tensor $\tilde{\sigma}$ is obtained from the linear superposition of the four relevant components considered in Fig. 10:

$$
\tilde{\sigma}=\sigma_{E 1} \mathbf{S}_{E 1}^{\prime}+\sigma_{G 1} \mathbf{S}_{G 1}^{\prime}+\sigma_{E 2} \mathbf{S}_{E 2}^{\prime}+\sigma_{G 2} \mathbf{S}_{G 2}^{\prime},
$$

where $\mathbf{S}_{\alpha i}^{\prime}$ are the Schmidt tensors that give the maximum resolved shear stress of each component on each plane. However, as defined in Eq. (10), the four tensors $\mathbf{S}_{\alpha i}^{\prime}$ will not necessarily be independent of one another and the application of one isolated component on any one plane may result in a resolved shear stress on the other. This is not in itself an erroneous proposition, as in reality any combination of stresses, independent or not, that produces the desired Peach-Köhler forces is valid. However, for simplicity and mathematical elegance it is best to find a solution for Eq. (9) that renders the $\mathbf{S}_{\alpha i}$ tensors independent from one another through a relation with the Schmidt tensors in Eq. (10). 


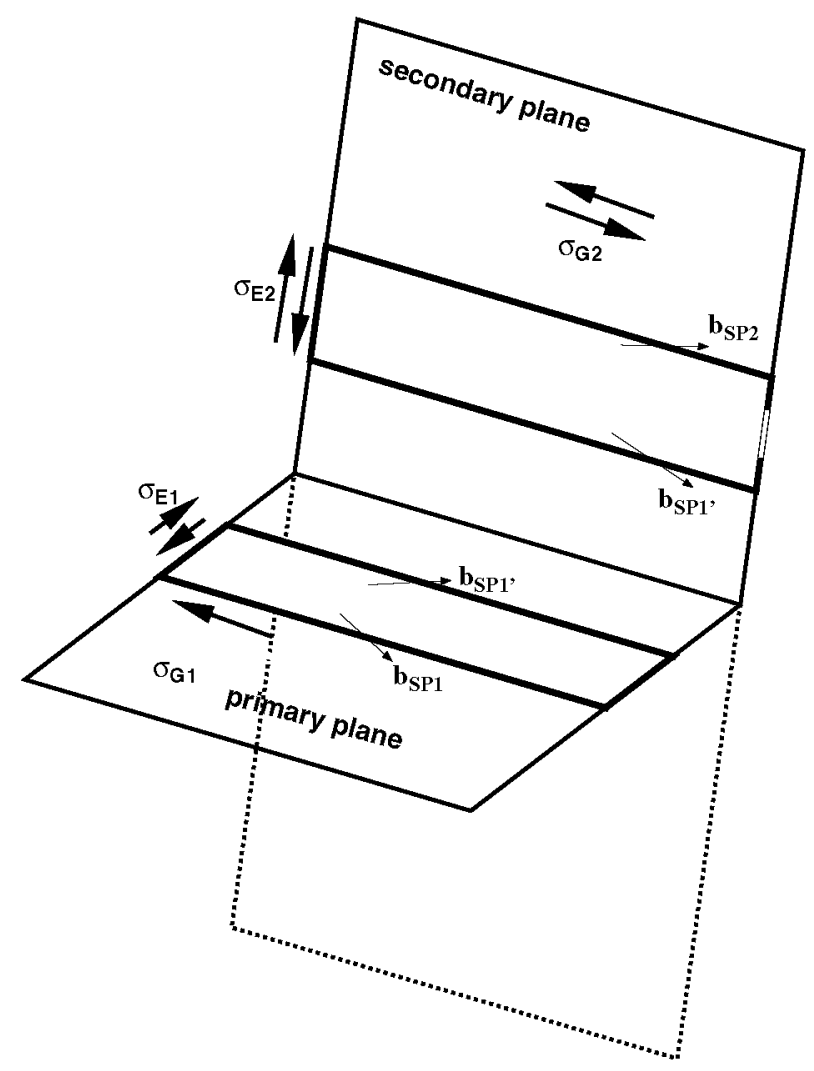

Fig. 10. Schematic diagram showing the geometry of the glide (primary) and cross-slip (secondary) planes with the four relevant components of the stress tensor, namely Escaig and glide stresses on both planes. Shown schematically on each plane are two extended screw dislocations. The shaded stripes are the stacking fault ribbons. Shockley partials are shown in black.

The relation between $\mathbf{S}_{\alpha i}^{\prime}$ and $\mathbf{S}_{\alpha i}$ simply follows from the imposition of orthogonality:

$$
\begin{aligned}
& \mathbf{S}_{\alpha i}^{\prime} \mathbf{S}_{\alpha i}=\mathbf{I}, \\
& \mathbf{S}_{\alpha i}^{\prime} \mathbf{S}_{\beta j}=0 .
\end{aligned}
$$

In this fashion, we ensure that $\sigma_{\alpha i}$ are mutually orthogonal and, thus, can be applied independently. Fig. 11 gives the direction of the forces derived from projecting these four relevant stresses on each plane.

Now, our intention is to explore the four-dimensional stress space defined by the four components in Eq. (9) in order to identify the threshold stress hypersurface that marks the transition from glide to cross-slip. Additionally, we are also interested in determining the pertinent cross-slip mechanism, as given in Section 3.4, under each stress condition. For simplicity, we study the projections of this four-dimensional stress space on the three-dimensional subspaces defined by the combinations of Escaig and glide stress on each plane: i.e. $\sigma_{G 1}-\sigma_{E 1}-\sigma_{G 2}, \sigma_{E 1}-\sigma_{G 2}-\sigma_{E 2}, \sigma_{G 1}-\sigma_{G 2}-\sigma_{E 2}$ and $\sigma_{G 1}-\sigma_{E 1}-\sigma_{E 2}$.

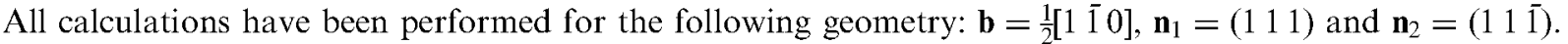
The length of the initial straight dislocation was of approximately $75 \mathrm{~nm}$. The value ranges for each of the stress components considered were determined from geometric stability conditions such as partial dislocation constriction or runaway partials.

Finally, we have calibrated the cross-slip maps from the minimum glide stress on the secondary plane required to induce cross-slip by a Fleischer mechanism in the absence of any Escaig stress on the primary plane $\left(\sigma_{E 1}=0, \sigma_{G 2}=2122.6 \mathrm{MPa}\right)$. This value is closely related to the energy required to nucleate a stair-rod dislocation in Fleischer's model. Both of these values have been obtained numerically using specifically 


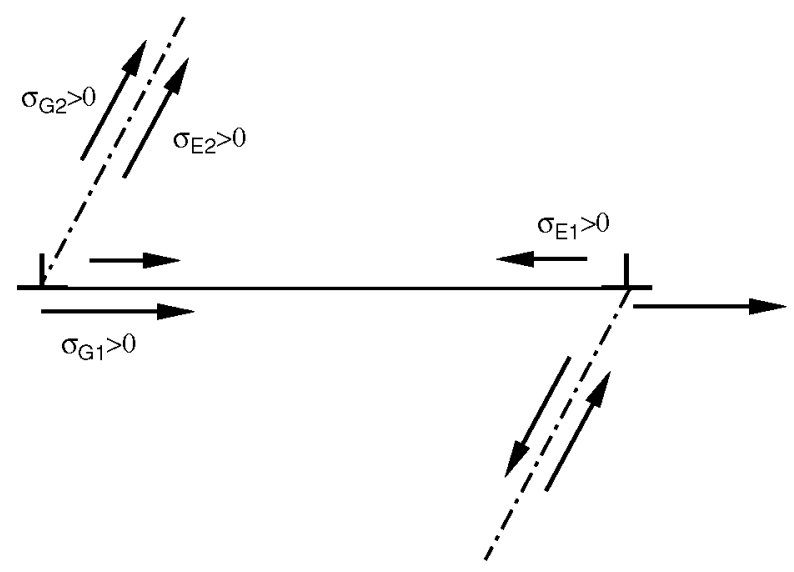

Fig. 11. Schematic view along the dislocation line of the different forces created by the four orthogonal stresses relevant for cross-slip. The direction of the forces shown for each Shockley partial correspond to positive values of the stress. As shown in the figure, in some instances, the stresses produce forces with opposite signs on different partials. This interplay among different stress components governs the cross-slip mechanisms and gives the stress maps shown below.

designed atomistic simulations and provide physically based input from which to build the stress maps. In what follows, we make use of this and the parametrization performed in Section 2.4 to construct the surfaces plotted in Figs. 12-15. More details about each one of them are given in each corresponding subsection below.

\subsubsection{Glide and Escaig stress on primary plane; Escaig stress on secondary plane}

The three-dimensional map for this stress combination is plotted in Fig. 12. The calculations were performed by relaxing the screw dislocation to the equilibrium distance dictated by the value of $\sigma_{E 1}$ and then applying pairs $\left(\sigma_{G 1}, \sigma_{E 2}\right)$ to obtain the surface points. The surface obtained (in color) represents the locus of the triad $\sigma_{G 1}-\sigma_{E 1}-\sigma_{E 2}$ above which the dislocation is seen to cross-slip by a Fleischer mechanism, i.e. no cross-slip occurs below the colored surface. $\sigma_{E 1}$ ranges from the value for runaway partials at $-0.0079 \mu$ to $0.0316 \mu$ at which full constriction is attained in the absence of any other stress components (see Section 2.4).

A salient characteristic of this stress subspace is the absence of cross-slip for negative Escaig stresses on the secondary plane. The reason is that, with the simulated geometry, only positive values of $\sigma_{E 2}$ will produce acute cross-slip. As we showed in Section 3.2.1, obtuse cross-slip is forbidden in our simulations due to, presumably, the high extrinsic stacking fault energy in our $\mathrm{Cu}$ model. Therefore, a positive or negative value for $\sigma_{E 2}$ must not be understood strictly in terms of constriction or separation of partials in this case, but, rather, as giving the direction of the force that produces cross-slip in the acute or obtuse sense. Because $\sigma_{E 2}$ acts upon both partials simultaneously and in the same fashion, cross-slip with these components always produces anti-symmetric structures such as those shown in Fig. 11. In this sense, the primordial effect of the glide stress on the primary plane is simply to facilitate or hamper (for small and large values of $\left|\sigma_{G l}\right|$, respectively) the onset of cross-slip in this subspace. Of course, the cross-slip map is perfectly symmetric with respect to any glide component on either plane, as in the starting configuration the two Shockley partials can be considered indistinguishable for all practical purposes.

The shaded boundary plane at $\sigma_{E 1}=0.0316 \mu$ shown in the figure effectively marks the transition from a Fleischer to a Friedel-Escaig cross-slip mechanism for any non-zero value of the stress components acting on the secondary plane. However, because the only component on the secondary plane considered for this subspace is $\sigma_{E 2}$ and, as we showed, this can only take positive values, in order to have Friedel-Escaig cross-slip it is actually required that $\sigma_{E 2}$ be positive. In other words, the mathematical condition that must be satisfied in this stress subspace to have Friedel-Escaig cross-slip is $\left(\sigma_{E 1} \geqslant 0.0316 \mu, \forall \sigma_{E 2}\right)$. 


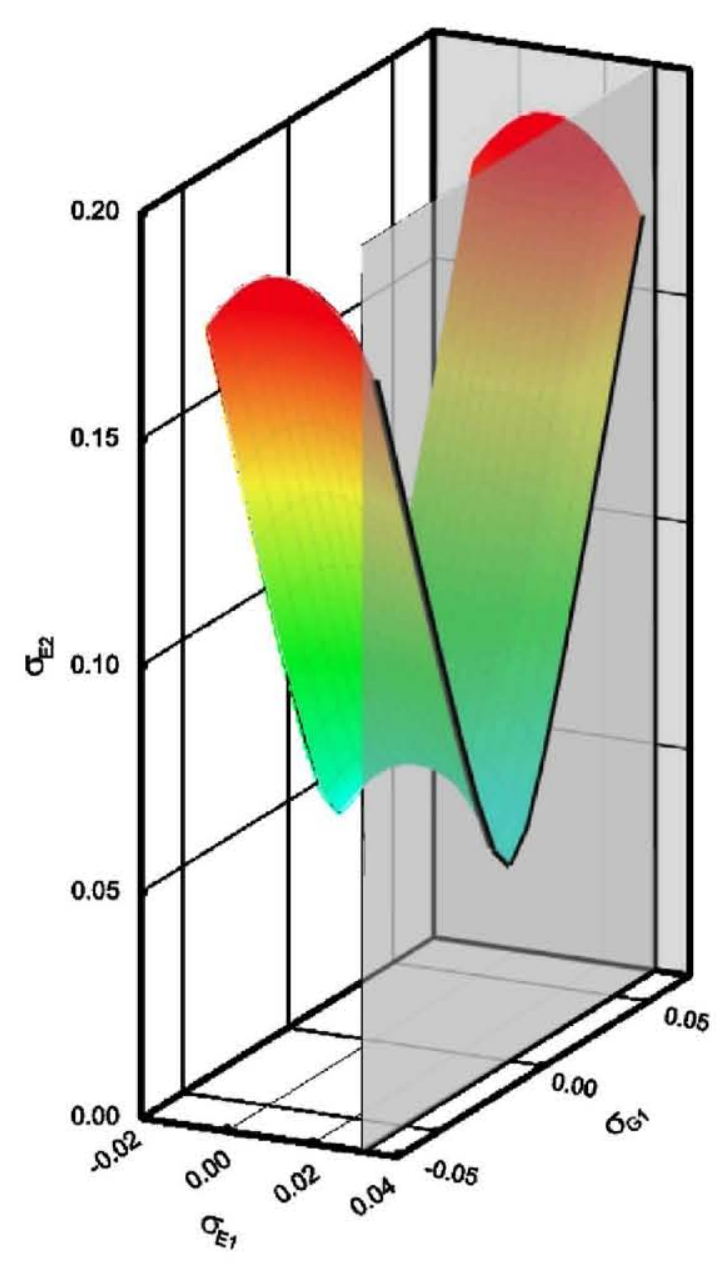

Fig. 12. Three-dimensional stress map for the cross-slip of a perfect screw dislocation from the primary to the secondary plane according to Fig. 10 when Escaig stress is applied on both planes and glide stress is only applied on the primary plane. All stresses are normalized to the value of the shear modulus $\mu$. Positive values of $\sigma_{E 1}$ or $\sigma_{E 2}$ indicate constriction stress. Note how $\sigma_{E 1}$ ranges from the value for runaway partials of $-0.0079 \mu$ to $0.0316 \mu$ at which full constriction is attained (shaded plane).

$\sigma_{E 2}$ shows a parabolic dependence with $\sigma_{E 1}$ with a maximum at a value of $\sigma_{E 1}=600 \mathrm{MPa}(0.0095 \mu)$. Presumably, this is the point at which the elastic force balance between the different dislocations involved is such that cross-slip is most hindered.

\subsubsection{Glide and Escaig stress on primary plane; glide stress on secondary plane}

As pointed out above, all solutions are symmetric with respect to either of the two glide stresses. This is clearly illustrated in Fig. 13, where both $\sigma_{G 1}=0$ and $\sigma_{G 2}=0$ are planes of mirror symmetry. In this case, the surfaces shown in the figure represent the loci $\sigma_{G 2}=f\left(\sigma_{G 1}, \sigma_{E 1}\right)$ above which cross-slip by a Fleischer mechanism occurs. Of course, by virtue of symmetry, the mathematical condition for cross-slip can simply be expressed as $\left|\sigma_{G 2}\right| \geqslant f\left(\left|\sigma_{G 1}\right|,\left|\sigma_{E 1}\right|\right)$, i.e. cross-slip takes place for stress triads on or above the red surface or on or below the blue surface.

Again in these calculations, there exists a maximum (in absolute value) $\sigma_{E 2}$ at the point where the elastic force balance is most unfavorable for cross-slip $\left(\sigma_{E 1}=0.0095 \mu\right)$. This is a somewhat puzzling observation, as one would think that the higher energy configurations induced by the Escaig stress might be relaxed by spreading on the cross-slip plane. However, we have checked this observation by simulating equivalent dislocation geometries using molecular dynamics and the atomistic results are consistent with the DD simulations: cross-slip is eased at primary Escaig stresses below and above $\sim 600 \mathrm{MPa}$. 

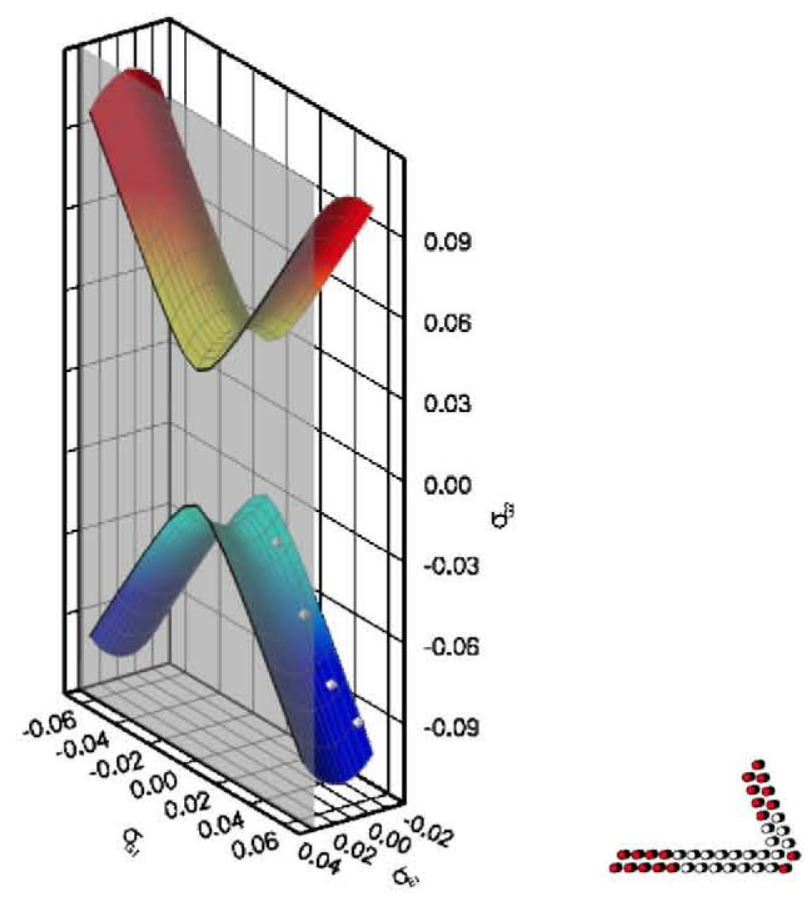

Fig. 13. Three-dimensional stress map for the cross-slip of a perfect screw dislocation from the primary to the secondary plane according to Fig. 10 when glide stress is applied on both planes and Escaig stress is only applied on the primary plane. All stresses are normalized to the value of the shear modulus $\mu$. The shaded plane marks the divide between cross-slip by Fleischer and Friedel-Escaig mechanisms. The light octahedral dots are equivalent MS results in the $\left(\sigma_{E 1}=0, \sigma_{G 1}>0, \sigma_{G 2}<0\right)$ stress quadrant. The inset shows an edge-on view of a screw dislocation undergoing cross-slip as obtained with molecular statics (red atoms are partial dislocations, white atoms belong to a stacking fault).

As for the previous case, the maximum value of $\sigma_{E 1}$ is that for which full partial dislocation constriction is attained and, hence, the plane $\sigma_{E 1}=0.0316 \mu$ marks the transition from Fleischer to Friedel-Escaig for any non-zero value of $\sigma_{G 2}$.

Validation of these simulations is an important aspect of this work. Consequently, for this particular stress space, we have chosen to explore the quadrant defined by $\left(\sigma_{E 1}=0, \sigma_{G 1}>0, \sigma_{G 2}<0\right)$ using MS simulations. The results appear as light octahedral dots in Fig. 13. The MS data are consistently higher (with errors ranging from $\sim 5 \%$ to $17 \%$ ) than the DD calculations, signaling the occurrence of cross-slip at lower stresses. Nevertheless, the trend followed by the atomistic results is in agreement with that displayed by DD. Although these results are preliminary, and suffer from the same limitations as those obtained in Section 2.4 using MS, they add confidence to our methodology. The inset in Fig. 13 shows an edge-on view of a screw dislocation undergoing cross-slip as obtained in our MS calculations.

\subsubsection{Escaig stress on primary plane; glide and Escaig stress on secondary plane}

The $\sigma_{E 1}-\sigma_{G 2}-\sigma_{E 2}$ map is plotted in Fig. 14. In this case, the curves exhibit only one symmetry plane $\left(\sigma_{G 2}=0\right)$, at which they intersect. This means that for certain combinations of $\sigma_{E 1}$ and $\sigma_{E 2}$ that satisfy the relation $\sigma_{E 2} \geqslant\left. f\left(\sigma_{E 1}\right)\right|_{\sigma_{G 2}=0}$, cross-slip by a Fleischer mechanism always occurs regardless of the value of $\sigma_{G 2}$.

The same effect observed in Section 3.4.1 regarding Escaig stress on the secondary plane is also seen here. The sign of $\sigma_{E 2}$ cannot be interpreted in terms of constriction/separation but rather as assisting in inducing acute or obtuse cross-slip. For acute cross-slip (the only one possible) to occur when $\sigma_{E 2}<0$, increasing amounts of glide stress on the secondary plane must be applied to overcome the obtuse cross-slip tendency created by the secondary Escaig stress. Consequently, the cross-slip forces on each partial due to the stress components acting on the secondary plane are additive in one case and opposing in the other, akin to the mechanism shown in Fig. 11. Therefore, the mathematical condition for cross-slip by a Fleischer mechanism is 


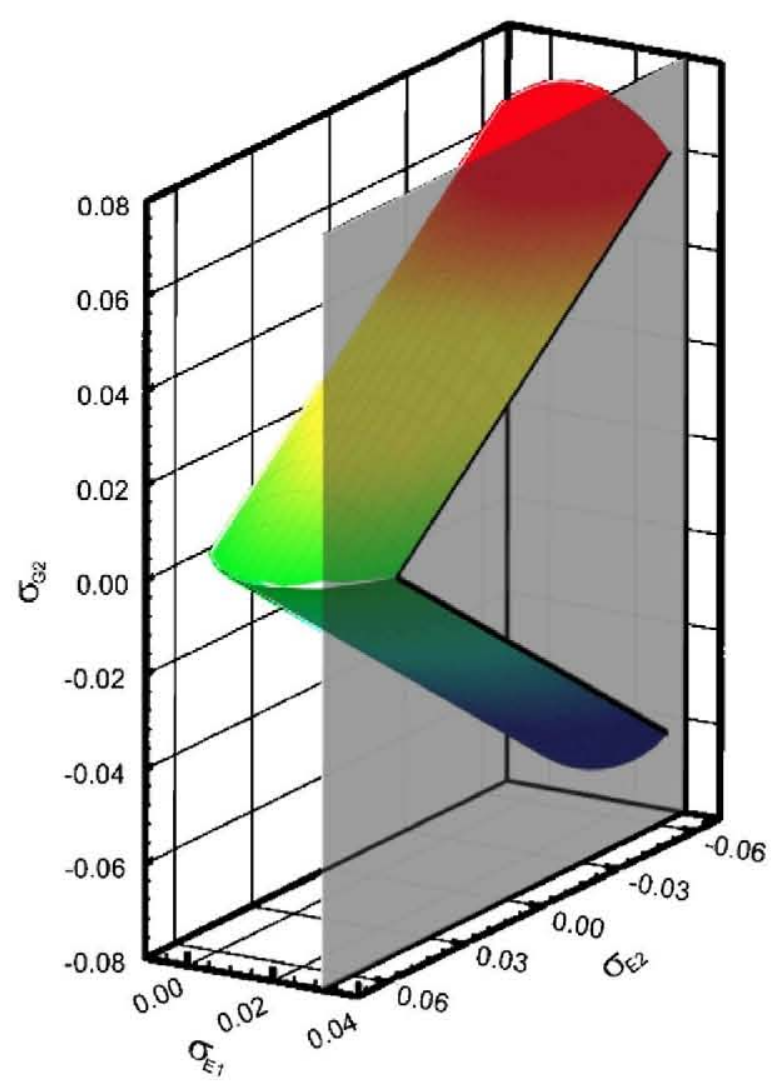

Fig. 14. Three-dimensional stress map for the cross-slip of a perfect screw dislocation from the primary to the secondary plane according to Fig. 10 when Escaig stress is applied on both planes and glide stress is only applied on the cross-slip plane. All stresses are normalized to the value of the shear modulus $\mu$. The shaded plane indicates the divide between cross-slip by Fleischer and Friedel-Escaig mechanisms.

simply $\left(\left|\sigma_{G 2}\right|+\sigma_{E 2}>0, \forall \sigma_{E 1}\right)$. Here we also capture the repulsive effect caused by constricted partials on cross-slipped configurations, i.e. the more we depart from the critical value of $\sigma_{E 1}=0.0095 \mu$, the lower the value of the glide stress on the secondary plane needed to provoke cross-slip. As expected from their colinearity, a linear relationship exists between $\sigma_{E 2}$ and $\sigma_{G 2}$.

Here too $\sigma_{E 1}$ ranges between $-0.0079 \mu$ and $0.0316 \mu$, beyond which, respectively, we have runaway partials and Friedel-Escaig cross-slip. The mathematical condition required for Friedel-Escaig cross-slip in this case is $\sigma_{E 1} \geqslant 0.0316 \mu, \forall \sigma_{G 2} \neq 0$.

\subsubsection{Glide stress on primary plane; glide and Escaig stress on secondary plane}

The stress surface corresponding to the $\sigma_{G 1}-\sigma_{G 2}-\sigma_{E 2}$ map is shown in Fig. 15. Here again two planes of symmetry exist, as corresponds to having both glide components active. With this stress combination, no cross-slip by a Friedel-Escaig mechanism can ever occur, as, in the absence of any $\sigma_{E 1}$, the orthogonality conditions (11) prevent any constricting stresses to develop on the primary plane.

In this case, the effect of having Escaig stress on the secondary plane is also to direct cross-slip in the acute $\left(\sigma_{E 2}>0\right)$ or obtuse $\left(\sigma_{E 2}<0\right)$ senses. Since obtuse cross-slip is forbidden, a negative $\sigma_{E 2}$ must be overcome by the secondary glide stress acting in the opposite direction so that acute cross-slip can occur. That is why the surfaces exhibit an increasing trend for negative values of the secondary Escaig stress. At $\left(\sigma_{G 1}, \sigma_{E 2}=0\right), \sigma_{G 2}$ takes the calibrated value of $2122.6 \mathrm{MPa}(0.034 \mu)$. With respect to the $\sigma_{G 1}-\sigma_{E 2}$ dependency, similar to the case in Section 3.4.1, there is only a very weak coupling between these two stress components.

Lastly, the $\sigma_{G 2}=f\left(\sigma_{G 1}\right)$ relation is of hyperbolic type, such that for increasingly larger values of $\left|\sigma_{G 1}\right|,\left|\sigma_{G 2}\right|$ must be increased to overcome the inertial resistance of the dislocation to cross-slip. As for the previous cases 


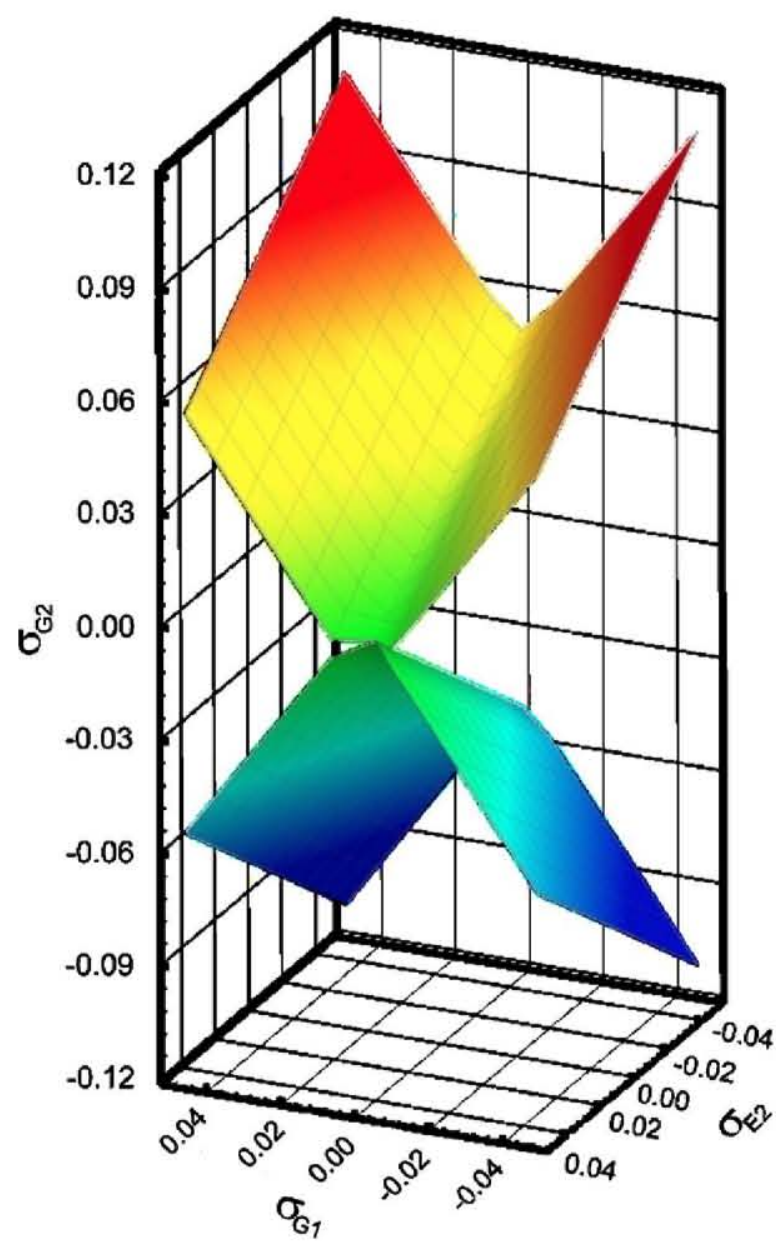

Fig. 15. Three-dimensional stress map for the cross-slip of a perfect screw dislocation from the primary to the secondary plane according to Fig. 10 when glide stress is applied on both planes and Escaig stress is only applied on the cross-slip plane. All stresses are normalized to the value of the shear modulus $\mu$.

where $\sigma_{E 2}$ is present, the cross-slip mechanism in this case is a combination of glide and Escaig on each partial, synergistic for one of them and competing for the other (Fig. 11).

\subsection{Discussion}

Below we briefly touch upon certain aspects of the methodology presented here. In this work, we have developed a DD model that takes into account the particular geometry of the fce lattice. Moreover, we have attempted to fit all the free parameters of the model $(\mu, \gamma$, Poisson's ratio, $v$, the dislocation mobility friction coefficients, $B$, and the dislocation core width, $a$, and energies, $\varepsilon$ ) using physically sound data, preferably obtained from the atomistic scale. It is worth emphasizing that the main purpose of this work is to present the methodology, its potential, and its limitations. Some useful checks for validation or application purposes have been provided, but more detailed studies related to fcc plasticity will be left for future publications. Let the examples presented in Section 3 suffice to highlight the capabilities of the method and provide simple and consistent validation checks. The central idea of our work is that a detailed DD methodology fitted to simple systems can be usefully applied to study complex systems.

As regards dislocation locks and forest hardening, the coefficients $\alpha_{i j}$ in Eq. (7) can either be calculated directly - by a number of different methods, or can be inferred from plasticity experiments and large-scale dislocation dynamics simulations. In this sense, our calculations in Section 3.2 suggest that, for the conditions 
chosen in our study (equi-stressed planes), the co-linear junction is the strongest, followed by the Lomer-Cottrell, and the Hirth locks. Nevertheless, the strength of a particular type of lock does not necessarily correlate with its relative importance in terms of plastic hardening. The appropriate method to establish the true importance of each junction is to separate their relative contribution to the total hardening (as measured via Eq. (7)) in large-scale DD plasticity simulations. This has been the approach employed by a number of workers, most notably Madec et al. (2003) and Devincre et al. (2006), who have come up with selfconsistent ways to differentiate among each fcc lock in their simulations. Their conclusions, interestingly, confirm the first-order assumption (which also emanates from our results) that there might be a direct correspondence between junction strength and their comparative contribution to hardening.

In conventional fcc plasticity, the rate of work hardening in stage II deformation, $\theta_{\mathrm{II}}$, is taken to be approximately universal and equal to $\sim \mu / 300$. These phenomenological models are formulated independently of the stacking fault energy of the material, or the zipping length over which the junction occurs, with reasonable agreement with experimental observations. What this suggests, indirectly, is that all that is needed to study stage II hardening are purely elastic models that neglect core effects and/or dislocation dissociation into partials. Our results, obtained incorporating core effects, do not disprove this extent, at least with the dislocation core model implemented here. The only way to shed more light on this issue is by performing studies of junction formation and dissolution with methods that resolve the dislocation core explicitly, e.g. methods capable of atomistic resolution.

Another interesting exercise is to further coarsen the different dislocation locks considered in Section 3.2 so that they can be expressed in terms of $\frac{1}{2}\langle 110\rangle$ dislocations. This may be useful in large plasticity simulations, where the detail of the reactions studied in Section 3.2 may lead to numerically intensive calculations and poor computational efficiency. The idea then would be to achieve self-consistency between the junctions obtained using the detailed partial dislocation model and the simplified perfect-dislocation approximation. As a preliminary example, we have computed the value of the core energy such that the strength of the Lomer-Cottrell lock studied in Section 3.2.1 matches the value given in Table 2. We have obtained a value of $4.11 \mathrm{eV} \mathrm{b}^{-1}$ for these conditions.

Regarding cross-slip, Figs. 13-15 show 'cold' stress maps, which provide a simple and effective criterion for cross-slip solely in terms of the remote stress state. However, in addition to their inherent numerical value, two important physics findings emanate from these stress maps. Firstly, we have discovered that there exists a value of constricting Escaig stress on the primary plane $(\sim 600 \mathrm{MPa})$ for which the dislocation ensemble is most unfavored to undergo cross-slip. We argue that this is due to the combination of elastic interactions among the partials involved. More calculations are needed, however, to prove or disprove this extent. Secondly, our results may narrow down the number of possible pathways for dislocation annihilation in Kubin et al.'s (2006) recent study on plastic recovery by removing the plausibility for obtuse cross-slip. As shown in Sections 3.2 and 3.4, we have demonstrated both using MS and DD simulations that, at least for $\mathrm{Cu}$, the energy cost of creating extrinsic faults precludes the formation of inverse junctions (e.g. obtuse Lomer-Cottrell or acute Hirth locks) and obtuse cross-slip. However, it is still difficult at this point to identify a direct means to validate our simulations, other than perhaps using atomistic results where available. In this sense, the comparison performed in Section 3.4.2 using atomistic data represents an encouraging quantitative and qualitative validation of our methodology, which makes use only of one stress data point as input. Ultimately, however, these stress maps will have to be incorporated into large-scale DD crystal plasticity simulations and the results benchmarked against existing experimental databases.

The remaining, and final, issue concerning cross-slip in fcc metals is to produce a full energy landscape of the cross-slip process as a function of stress so that thermal effects can be incorporated into the simulations. This can in principle be achieved by way of atomistic calculations (the atomistic works referenced in the previous paragraphs are essentially zero-stress calculations of these energies), although the associated computational cost can be daunting, or, provided that core energies and radii are properly accounted for, using numerically light elastic models in the fashion of Puschl (2002) to calculate configuration energies as a function of dislocation position and character. Once these energy barriers are known, one can use simple kinkpair/cross-slip models such as that devised by Rhee et al. (1998) or Khraishi et al. (2002) to study temperature effects on plasticity. 


\section{Conclusions}

In summary, we have extended the discrete DD methodology as developed by Arsenlis et al. (2007) and Bulatov et al. (2004) to fec systems by explicitly considering all dislocation dissociations and reactions among partials. To this end, we have derived simple continuity laws that enable the treatment of stacking faults and partial dislocation nodes. In addition, we have added appropriate topological rules to account for the specific transformations relevant to fcc slip, e.g. dislocation dissociation, junction formation, cross-slip, etc. Our DD methodology has been fitted to atomistic results in $\mathrm{Cu}$, including core energetics, and has withstood the test of several simple validation checks. We show that, consistent with real crystals, the sole parameter needed to describe all dislocation dissociations and reactions is the stacking fault energy.

We have obtained the dissolution strengths of the main four dislocation junctions in fcc metals, namely the Lomer-Cottrell, Hirth, co-linear and co-planar junctions, for the straightforward geometry of perfectly parallel initial dislocation segments. We find that, in agreement with the current state-of-the-art in DD simulations, the co-linear junction is clearly the strongest. A full mapping of the junction strength as a function of the character of the reacting dislocations will be undertaken in future studies.

We have characterized the four-dimensional cross-slip surface by mapping threefold stress subspaces and have extracted simple mathematical rules to implement the different cross-slip mechanisms considered. Based on atomistic simulations, our model does not include cross-slip into obtuse planes, as the stress required for this process is significantly larger than for the acute case. The thermally activated nature of cross-slip has not been treated here and our stress surfaces provide a cross-slip map in zero-temperature equivalent conditions. Future work includes computing the cross-slip energy landscape as a function of the applied stress so that thermal effects can be accounted for.

\section{Appendix A. Rotation of the stiffness tensor}

The components of the 4th rank elastic stiffness tensor $\mathbf{C}$ are readily transformed from the canonical frame of reference into a rotated frame of reference by

$$
c_{i j k l}^{\prime}=T_{i g} T_{j h} c_{g h m n} T_{k m} T_{l n},
$$

where the $T_{\alpha \beta}$ are the components of the rotation matrix $\mathbf{T}$ and represent the direction cosine between the rotated $x_{\alpha}^{\prime}$ and the initial $x_{\beta}$ axes. In our case we want to particularize $\mathbf{C}$, given by Mishin et al. (2001) for the

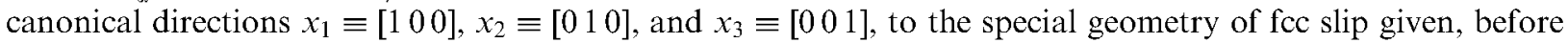

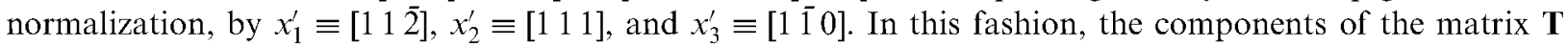
take the form:

$$
T_{\alpha \beta}=\frac{\mathbf{x}_{\alpha}^{\prime} \cdot \mathbf{x}_{\beta}}{\left|\mathbf{x}_{\alpha}^{\prime}\right|}
$$

i.e.,

$$
\mathbf{T}=\left[\begin{array}{ccc}
\frac{1}{\sqrt{6}} & \frac{1}{\sqrt{6}} & -\frac{2}{\sqrt{6}} \\
\frac{1}{\sqrt{3}} & \frac{1}{\sqrt{3}} & \frac{1}{\sqrt{3}} \\
\frac{1}{\sqrt{2}} & -\frac{1}{\sqrt{2}} & 0
\end{array}\right]
$$


Transformations are performed most conveniently in the complete $9 \times 9$ frame for $\mathbf{C}$, which, after symmetry reduction, is

$$
\mathbf{C}=\left[\begin{array}{ccccccccc}
c_{1111} & c_{1122} & c_{1122} & 0 & 0 & 0 & 0 & 0 & 0 \\
c_{1122} & c_{1111} & c_{1122} & 0 & 0 & 0 & 0 & 0 & 0 \\
c_{1122} & c_{1122} & c_{1111} & 0 & 0 & 0 & 0 & 0 & 0 \\
0 & 0 & 0 & c_{2323} & 0 & 0 & c_{2323} & 0 & 0 \\
0 & 0 & 0 & 0 & c_{2323} & 0 & 0 & c_{2323} & 0 \\
0 & 0 & 0 & 0 & 0 & c_{2323} & 0 & 0 & c_{2323} \\
0 & 0 & 0 & c_{2323} & 0 & 0 & c_{2323} & 0 & 0 \\
0 & 0 & 0 & 0 & c_{2323} & 0 & 0 & c_{2323} & 0 \\
0 & 0 & 0 & 0 & 0 & c_{2323} & 0 & 0 & c_{2323}
\end{array}\right],
$$

where, of course, in contracted matrix notation, $c_{1111}=C_{11}=169.9 \mathrm{MPa}, c_{1122}=C_{12}=122.6 \mathrm{MPa}$, and $c_{2323}=C_{44}=76.2 \mathrm{MPa}$, as given by Mishin et al. (2001).

Inserting Eqs. (A.3) and (A.4) into Eq. (A.1), one obtains a value of $C_{44}^{\prime}=63.2 \mathrm{MPa}$. In isotropic or lowanisotropy materials this value can be taken as the shear modulus $\mu$.

\section{References}

Amodeo, R.J., Ghoniem, N.M., 1991. In: Lowe, T., Rollett, T., Follansbee, P., Daehn, G. (Eds.), Modelling of Deformation of Crystalline Solids. TMS Press, Warrendale, PA, 1991, p. 125.

Arsenlis, A., Cai, W., Tang, M., Rhee, M., Oppelstrup, T., Hiratani, M., Hommes, G., Pierce, T.G., Bulatov, V.V., 2007. Modelling Simulations Mater. Sci. Eng., in press.

Basinski, S.J., Basinski, Z.S., 1979. In:Nabarro, F.R.N. (Ed.), Dislocations in Solids, vol. 4. North-Holland, Amsterdam, pp. $261-362$.

Bonneville, J., Escaig, B., 1979. Acta Metall. 27, 1477.

Bonneville, J., Vanderschaeve, G., 1998. Philos. Mag. Lett. 78, 87

Bonneville, J., Escaig, B., Martin, J.L., 1999. Acta Metall. 36, 1989

Bulatov, V.V., Yip, S., Argon, A.S., 1995. Philos. Mag. A 72, 453.

Bulatov, V.V., Tang, M.J., Zbib, H.M., 2001. MRS Bull. 26, 191.

Bulatov, V.V., Cai, W., Fier, J., Hiratani, M., Pierce, T., Tang, M., Rhee, M., Yates, K., Arsenlis, A., 2004. Scalable line dynamics of ParaDiS, SuperComputing 19, 〈http://www.sc-conference.org/sc2004/schedule/pdfs/pap206.pdf .

Cai, W., Bulatov, V.V., Chan, J., Li, J., Yip, S., 2001. Phys. Rev. Lett. 86, 5727.

Cai, W., Bulatov, V.V., Pierce, T.G., Hiratani, M., Rhee, M., Bartelt, M., Tang, M., 2004. Massively-parallel dislocation dynamics simulations. In: Kitagawa, H., Shibutani, Y. (Eds.), Solid Mechanics and its Applications. Kluwer Academic Publisher, Dordrecht. Cai, W., Arsenlis, A., Weinberger, C.R., Bulatov, V.V., 2006. J. Mech. Phys. Solids 54, 561.

Caillard, D., Martin, J.L., 1989. J. Phys. (France) 50, 2455.

Caillard, D., Martin, R., 2003. Thermally Activated Mechanisms in Crystal Plasticity. Pergamon, Oxford, UK.

de Koning, M., Cai, W., Bulatov, V.V., 2003. Phys. Rev. Lett. 91, 025503.

Depres, C., Fivel, M., Robertson, C., Fissolo, A., Verdier, M., 2003. J. Phys. IV 106, 81.

Devincre, B., Kubin, L.P., 1997. Mater. Sci. Eng. A 8, 234.

Devincre, B., Kubin, L., Hoc, T., 2006. Scr. Mater. 54, 741.

Duesbery, M.S., 1998. Modelling Simulations Mater. Sci. Eng. 6, 35.

Duesbery, M.S., Louat, N.P., Sadananda, K., 1992a. Philos. Mag. 65, 311.

Duesbery, M.S., Louat, N.P., Sadananda, K., 1992b. Acta Metall. Mater. 40, 149.

Dupuy, L., Fivel, M.C., 2002. Acta Mater. 50, 4873.

Escaig, B., 1968. J. Phys. 29, 225.

Fleischer, R.L., 1959. Acta Metall. 7, 134.

Franciosi, P., Berveiller, M., Zaoui, A., 1980. Acta Metallurgica 28, 273.

Ghoniem, N.M., Amodeo, R., 1988. Solid State Phenom. 3\&4, 377.

Ghoniem, N.M., Sun, L.Z., 1999. Phys. Rev. B 60, 128.

Hardikar, K., Shenoy, V., Phillips, R., 2001. J. Mech. Phys. Solids 49, 1951.

Henager, C.H., Hoagland, R.G., 2005. Philos. Mag. 85, 4477.

Hirth, J.P., 1961. J. Appl. Phys. 32, 700.

Hirth, J.P., Lothe, J., 1992. Theory of Dislocations. Krieger, Malabar, Florida, p. 345.

Jackson, P.J., 1984. Prog. Mater. Sci. 29, 139. 
Justo, J.F., Bulatov, V.V., Yip, S., 1997. Scr. Mater. 36, 707.

Khraishi, T.A., Zbib, H.M., Diaz de la Rubia, T., Victoria, M., 2002. Metall. Mater. Trans. B 33B, 285.

Kubin, L.P., Canova, G., Conat, M., Devincre, B., Pontikis, V., Brechet, Y., 1992. Solid State Phenom. 23 \& 24, 455.

Kubin, L.P., Devincre, B., Tang, M., 1998. J. Comput. Aided Mater. Des. 5, 31.

Kubin, L.P., Madec, R., Devincre, B., 2003. In: Multiscale Phenomena in Materials - Experiments and Modeling Related to Mechanical Behavior, Material Research Society Symposium Proceedings, vol. 779. Warrendale, PA, USA, p. 25.

Kubin, L.P., Devincre, B., Hoc, T., 2006. Philos. Mag. 86, 4023.

Lee, H.J., Wirth, B.D., et al., 2007. private communication.

Lepinoux, J., Kubin, L.P., 1987. Scr. Metall. 21, 833.

LeSar, R., 2004. Phys. Status Solidi B 241, 2875.

Li, J., Wang, C.-Z., Chang, J.-P., Cai, W., Bulatov, V.V., Ho, K.-M., Yip, S., 2004. Phys. Rev. B 70, 104113.

Lothe, J., Hirth, J.P., 2005. Phys. Status Solidi B 242, 836.

Madec, R., Devincre, B., Kubin, L.P., 2002. Phys. Rev. Lett. 89, 255508.

Madec, R., Devincre, B., Kubin, L., Hoc, T., Rodney, D., 2003. Science 301, 1879.

Marian, J., Caro, A., 2006. Phys. Rev. B 74, 024113.

Mishin, Y., Mehl, M.J., Papaconstantopoulos, D.A., Voter, A.F., Kress, J.D., 2001. Phys. Rev. B 63, 224106.

Mohles, V., 2001. Mater. Sci. Eng. A 319-321, 206.

Mohles, V., 2002. Mater. Sci. Eng. A 324, 190.

Mordehai, D., Kelson, I., Makov, G., 2005. Mater. Sci. Eng. A 400-401, 37.

Pendurti, S., Jun, S., Lee, I.-H., Prasad, V., 2006. Appl. Phys. Lett. 88, 201908.

Puschl, W., 2002. Prog. Mater. Sci. 47, 415.

Rao, S., Parthasarathy, T.A., Woodward, C., 1999. Philos. Mag. A 79, 1167.

Rasmussen, T., Jacobsen, W.K., Leffers, T., Pedersen, O.B., Srinivasan, S.G., Jonsson, H., 1997a. Phys. Rev. Lett. $79,3676$.

Rasmussen, T., Jacobsen, W.K., Leffers, T., Pedersen, O.B., 1997b. Phys. Rev. B 56, 2997.

Rhee, M., Zbib, H.M., Hirth, J.P., Huang, H., de la Rubia, T., 1998. Modelling Simulations Mater. Sci. Eng. 6, 467.

Rodney, D., Phillips, R., 1999. Phys. Rev. Lett. 82, 1704.

Schwarz, K.W., 1999. J. Appl. Phys. 85, 108.

Shehadeh, M.A., Zbib, H.M., de la Rubia, T.D., 2005. Philos. Mag. 85, 1667.

Shenoy, V.B., Kukta, R.V., Phillips, R., 2000. Phys. Rev. Lett. 84, 1491.

Shin, C.S., Shivel, M.C., Rodney, D., Phillips, R., Shenoy, V.B., Dupuy, L., 2001. J. Phys. IV France 11, 5.

Shoeck, G., Seeger, A., 1955. Defects in Crystalline Solids. The Physical Society, London, p. 340.

Sun, Y.Q., Hazzledine, P.M., Crimp, M.A., Couret, A., 1991. Philos. Mag. A 34, 311.

Vegge, T., Jacobsen, K.W., 2002. J. Phys. Condens. Matter 4, 2929.

Verdier, M., Fivel, M., Groma, I., 1998. Modelling Simulations Mater. Sci. Eng. 6, 755.

Vitek, V., 1985. Trans. Indian Inst. Met. 38, 510.

Wang, Z., McCabe, R.J., Ghoniem, N.M., LeSar, R., Misra, A., Mitchell, T.E., 2004. Acta Mater. 52, 1535.

Zbib, H.M., Diaz de la Rubia, T., 2002. Int. J. Plasticity 18, 1133.

Zbib, H.M., Rhee, M., Hirth, J.P., 1998. Int. J. Mech. Sci. 40, 113.

Zbib, H.M., Diaz de la Rubia, T., Rhee, M., Hirth, J.P., 2000. J. Nucl. Mater. 276, 154.

Zhou, S.J., Preston, D.L., Lomdahl, P.S., Beazley, D.M., 1998. Science 279, 1525. 